\title{
ANTICHAINS FOR THE AUTOMATA-BASED APPROACH TO MODEL-CHECKING*
}

\author{
LAURENT DOYEN $^{a}$ AND JEAN-FRANÇOIS RASKIN ${ }^{b}$
}

${ }^{a}$ CCS, École Polytechnique Fédérale de Lausanne, Switzerland

e-mail address: laurent.doyen@epfl.ch

${ }^{b}$ CS, Université Libre de Bruxelles, Belgium

e-mail address: jraskin@ulb.ac.be

\begin{abstract}
We propose and evaluate antichain algorithms to solve the universality and language inclusion problems for nondeterministic Büchi automata, and the emptiness problem for alternating Büchi automata. To obtain those algorithms, we establish the existence of simulation pre-orders that can be exploited to efficiently evaluate fixed points on the automata defined during the complementation step (that we keep implicit in our approach). We evaluate the performance of the algorithm to check the universality of Büchi automata using the random automaton model recently proposed by Tabakov and Vardi. We show that on the difficult instances of this probabilistic model, our algorithm outperforms the standard ones by several orders of magnitude.
\end{abstract}

\section{INTRODUCTION}

In the automata-based approach to model-checking [VW86, VW94, programs and properties are modeled by finite automata. Let $A$ be a finite automaton that models a program and let $B$ be a finite automaton that models a specification that the program should satisfy. Correctness is defined by the language inclusion $\mathcal{L}(A) \subseteq \mathcal{L}(B)$, that is all traces of the program (executions) should be traces of the specification. To solve the inclusion problem, the classical automata-theoretic solution constructs an automaton for $\mathcal{L}^{c}(B)$ the complement of the language of the automaton $B$ and then checks that $\mathcal{L}(A) \cap \mathcal{L}^{c}(B)$ is empty (the later intersection being computed as a synchronised product).

1998 ACM Subject Classification: F.4.1, I.1.2.

Key words and phrases: alternating Büchi automata, nondeterministic Büchi automata, emptiness, universality, language inclusion, antichains.

* A preliminary version of this paper appeared in the Proceedings of the 13th International Conference on Tools and Algorithms for the Construction and Analysis of Systems (TACAS), Lecture Notes in Computer Science 4424, Springer-Verlag, 2007, pp. 451-465.

This research was supported in part by the FRFC project "Centre Fédéré en Vérification" funded by the Belgian National Science Foundation (FNRS) under grant nr 2.4530.02, by the PAI program Moves supported by the Belgian Federal Gouvernment: "Fundamental Issues in Modelling, Verification and Evolution of Software" (http://moves.vub.ac.be), by the Swiss National Science Foundation, and by the European COMBEST project. 
In the finite case, the program and the specification are automata over finite words (NFA) and the construction for the complementation is conceptually simple: it is achieved by a classical subset construction. In the case of infinite words, the program and (or at least) the specification are nondeterministic Büchi automata (NBW). The NBW are also complementable; this was first proved by Büchi Büc62. However, the result is much harder to obtain than in the case of NFA. The original construction of Büchi has a $2^{O\left(2^{n}\right)}$ worst case complexity (where $n$ is the size of the automaton to complement) which is not optimal. In the late eighties Safra in [Saf88, and later Kupferman and Vardi in [KV01, have given optimal complementation procedures that have $2^{O(n \log n)}$ complexity (see [Mic88] for the lower bound). While for finite words, the classical algorithm has been implemented and shown practically usable, for infinite words, the theoretically optimal solution is difficult to implement and very few results are known about their practical behavior. Recent implementations have shown that applying these algorithms for automata with more than around ten states is hard [TV07, GKSV03]. Such sizes are clearly not sufficient in practice. As a consequence, tools like SPIN [RH04 that implement the automata-theoretic approach to model-checking ask either that the complement of the specification is explicitly given or they limit the specification to properties that are expressible in LTL.

In this paper, we propose a new approach to check $\mathcal{L}(A) \subseteq \mathcal{L}(B)$ that can handle much larger Büchi automata. In a recent paper, we have shown that the classical subset construction can be avoided and kept implicit for checking language inclusion and language universality for NFA and their alternating extensions [DDHR06]. Here, we adapt and extend that technique to the more intricate case of automata on infinite words.

To present the intuition behind our new techniques, let us consider a simpler setting of the problem. Assume that we are given a NBW $B$ and we want to check if $\Sigma^{\omega} \subseteq \mathcal{L}(B)$, that is to check if $\mathcal{L}(B)$ is universal. First, remember that $\mathcal{L}(B)$ is universal when its complement $\mathcal{L}^{c}(B)$ is empty. The classical algorithm first complements $B$ and then checks for emptiness. The language of a NBW is nonempty if there exists an infinite run of the automaton that visits accepting locations infinitely often. The existence of such a run can be established in polynomial time by computing the following fixed point $\mathcal{F} \equiv \nu y \cdot \mu x \cdot(\operatorname{Pre}(x) \cup(\operatorname{Pre}(y) \cap \alpha))$ where Pre is the predecessor operator of the automaton (given a set $L$ of locations it returns the set of locations that can reach $L$ in one step) and $\alpha$ is the set of accepting locations of the automaton. The automaton is non-empty if and only if its initial location is a member of the fixed point $\mathcal{F}$. This well-known algorithm is quadratic in the size of the automaton. Unfortunately, the automaton that accepts the language $\mathcal{L}^{c}(B)$ is usually huge and the evaluation of the fixed point is unfeasible for all but the smallest specifications $B$. To overcome this difficulty, we make the following observation: if $\preceq$ is a simulation pre-order on the locations of $B^{c}\left(\ell_{1} \preceq \ell_{2}\right.$ means $\ell_{1}$ can simulate $\left.\ell_{2}\right)$ which is compatible with the accepting condition (if $\ell_{1} \preceq \ell_{2}$ and $\ell_{2} \in \alpha$ then $\ell_{1} \in \alpha$ ), then the sets that are computed during the evaluation of $\mathcal{F}$ are all $\preceq$-downward-closed (if an element $\ell$ is in the set then all $\ell^{\prime} \preceq \ell$ are also in the set). Then $\preceq$-downward-closed sets can be represented by their $\preceq$-maximal elements and if operations on such sets can be computed directly on their representation, we have the ingredients to evaluate the fixed point in a more efficient way. For an automaton $\mathcal{B}$ over finite words, set inclusion would be a typical example of a simulation relation for $\mathcal{B}^{c}$ [DDHR06]. The same technique can be applied to avoid subset constructions in games of imperfect information [DDR06, CDHR07]. We generically call antichain algorithms the techniques that are based on compact representation of downward-closed because when the simulation 
is a partial order (and it usually is), the maximal elements form an antichain, i.e., a set of incomparable elements.

We show that the classical constructions for Büchi automata that are used in the automata-theoretic approach to model-checking are all equipped with a simulation preorder that exists by construction and does not need to be computed. On that basis we propose antichain algorithms to check universality of NBW, language inclusion for NBW, and emptiness of alternating Büchi automata (ABW). Each of these problems reduces to emptiness checking of NBW, via classcial constructions.

The novelty of our antichain algorithms is to realize that only downward-closed sets can be computed by the fixed point for emptiness, and therefore to use more succinct representations of those downward-closed sets, by storing maximal elements only. Moreover, such compact representations do not come at the price of an increase in the time complexity for the basic operations that are necessary to check emptiness (such as $\cap, \cup$, and Pre), i.e., we show that they are computable in time polynomial in the size of the compact representation, while this size can be exponentially smaller than the actual downward-closed set. Note that, while a compact representation exists in general (i.e., for any simulation pre-order), we have no generic result that would show that efficient computations can be done symbolically in all cases. Therefore, we have to instantiate the approach for each class of problem, and find efficient algorithms for the basic operations.

We evaluate an implementation of our algorithm for the universality problem of NBW and on a randomized model recently proposed by Tabakov and Vardi. We show that the performance of the antichain algorithm on this randomized model outperforms by several order of magnitude the existing implementations of the Kupferman-Vardi algorithm [TV07, GKSV03. While the classical solution is limited to automata of size 8 for some parameter values of the randomized model, we are able to handle automata with more than one hundred locations for the same parameter values. We have identified the hardest instances of the randomized model for our algorithms and show that we can still handle problems with several dozens of locations for those instances.

Structure of the paper. In Section 2, we give all necessary definitions related to Büchi automata, and we recall the Kupferman-Vardi and Miyano-Hayashi constructions that are used for complementation of NBW. The reader interested in the general theory behind our technique can read Section 3 without going into the details of those constructions (only the definitions of NBW and emptiness are useful to understand Section 3). The notion of simulation pre-order for a Büchi automaton is presented and we prove that the fixed point needed to establish emptiness of nondeterministic Büchi automata handles only downward closed sets for such pre-orders. We use this observation in Section 4 to define an antichain algorithm to decide emptiness of ABW. In Section 5, we adapt the technique for the universality problem of NBW. In Section 6 we report on the performances of the algorithm for universality, and in Section 7 , we extend those ideas to obtain an antichain algorithm for language inclusion of NBW.

\section{Büchi Automata and Classical Algorithms}

Definition 2.1. An alternating Büchi automaton (ABW) is a tuple $\mathcal{A}=\langle$ Loc, $\iota, \Sigma, \delta, \alpha\rangle$ where:

- Loc is a finite set of states (or locations). The size of $\mathcal{A}$ is $|\mathcal{A}|=\mid$ Loc $\mid$; 
- $\iota \in$ Loc is the initial state;

- $\Sigma$ is a finite alphabet;

- $\delta$ : Loc $\times \Sigma \rightarrow \mathcal{B}^{+}$(Loc) is the transition function where $\mathcal{B}^{+}$(Loc) is the set of positive boolean formulas over Loc, i.e. formulas built from elements in Loc $\cup$ true, false $\}$ using the boolean connectives $\wedge$ and $\vee$;

- $\alpha \subseteq$ Loc is the set of accepting states.

We say that a set $X \subseteq$ Loc satisfies a formula $\varphi \in \mathcal{B}^{+}$(Loc) (noted $X \models \varphi$ ) iff the truth assignment that assigns true to the members of $X$ and assigns false to the members of Loc $\backslash X$ satisfies $\varphi$. A run of $\mathcal{A}$ on an infinite word $w=\sigma_{0} \cdot \sigma_{1} \ldots$ is a DAG $T_{w}=\left\langle V, v_{\iota}, \rightarrow\right\rangle$ where:

- $V=\operatorname{Loc} \times \mathbb{N}$ is the set of nodes. A node $(\ell, i)$ represents the state $\ell$ after the first $i$ letters of the word $w$ have been read by $\mathcal{A}$. Nodes of the form $(\ell, i)$ with $\ell \in \alpha$ are called $\alpha$-nodes;

- $v_{\iota}=(\iota, 0) \in V$ is the root of the DAG;

- and $\rightarrow \subseteq V \times V$ is such that $(i)$ if $(\ell, i) \rightarrow\left(\ell^{\prime}, i^{\prime}\right)$ then $i^{\prime}=i+1$ and $(i i)$ for every $(\ell, i) \in V$, the set $\left\{\ell^{\prime} \mid(\ell, i) \rightarrow\left(\ell^{\prime}, i+1\right)\right\}$ satisfies the formula $\delta\left(\ell, \sigma_{i}\right)$.

We say that $\left(\ell^{\prime}, i+1\right)$ is a successor of $(\ell, i)$ if $(\ell, i) \rightarrow\left(\ell^{\prime}, i+1\right)$, and we say that $\left(\ell^{\prime}, i^{\prime}\right)$ is reachable from $(\ell, i)$ if $(\ell, i) \rightarrow^{*}\left(\ell^{\prime}, i^{\prime}\right)$.

A run $T_{w}=\left\langle V, v_{\iota}, \rightarrow\right\rangle$ of $\mathcal{A}$ on an infinite word $w$ is accepting iff all its infinite paths $\pi$ rooted at $v_{\iota}$ visit $\alpha$-nodes infinitely often. An infinite word $w \in \Sigma^{\omega}$ is accepted by $\mathcal{A}$ if there exists an accepting run on it. We denote by $\mathcal{L}(\mathcal{A})$ the set of infinite words accepted by $\mathcal{A}$, and by $\mathcal{L}^{c}(\mathcal{A})$ the set of infinite words that are not accepted by $\mathcal{A}$.

Definition 2.2. A nondeterministic Büchi automaton (NBW) is an ABW whose transition function is restricted to disjunctions over Loc.

Runs of NBW reduce to (linear) traces. The transition function of NBW is often seen as a function $\left[Q \times \Sigma \rightarrow 2^{Q}\right]$ and we write $\delta(\ell, \sigma)=\left\{\ell_{1}, \ldots, \ell_{n}\right\}$ instead of $\delta(\ell, \sigma)=$ $\ell_{1} \vee \ell_{2} \vee \cdots \vee \ell_{n}$. We note by $\operatorname{Pre}_{\sigma}^{\mathcal{A}}(L)$ the set of predecessors by $\sigma$ of the set $L$ : $\operatorname{Pre}_{\sigma}^{\mathcal{A}}(L)=$ $\left\{\ell \in \operatorname{Loc} \mid \exists \ell^{\prime} \in L: \ell^{\prime} \in \delta(\ell, \sigma)\right\}$. Let $\operatorname{Pre}^{\mathcal{A}}(L)=\left\{\ell \in \operatorname{Loc} \mid \exists \sigma \in \Sigma: \ell \in \operatorname{Pre}_{\sigma}^{\mathcal{A}}(L)\right\}$.

Problems. The emptiness problem for NBW is to decide, given an NBW $\mathcal{A}$, whether $\mathcal{L}(\mathcal{A})=\emptyset$. This problem is solvable in polynomial time. The symbolic approach through fixed point computation is quadratic in the size of $\mathcal{A}$ [EL86]. Other symbolic approaches have been proposed with better complexity bounds [BGS00, GPP03, but the fixed point computation shows better performances in practice RBS00.

The universality problem for NBW is to decide, given an NBW $\mathcal{A}$ over the alphabet $\Sigma$ whether $\mathcal{L}(\mathcal{A})=\Sigma^{\omega}$ where $\Sigma^{\omega}$ is the set of all infinite words on $\Sigma$. This problem is PSPACEcomplete [SVW87]. The classical algorithm to decide universality is to first complement the NBW and then to check emptiness of the complement. The difficult step is the complementation as it may cause an exponential blow-up in the size of the automaton. There exist two types of construction, one is based on a determinization of the automaton [Saf88] and the other uses ABW as an intermediate step [KV01]. We review the second construction below.

The language inclusion problem for NBW is to decide, given two NBW $\mathcal{A}$ and $\mathcal{B}$, whether $\mathcal{L}(\mathcal{A}) \subseteq \mathcal{L}(\mathcal{B})$. This problem is central in model-checking and it is PSPACE-complete in the size of $\mathcal{B}$. The classical solution consists in checking the emptiness of $\mathcal{L}(\mathcal{A}) \cap \mathcal{L}^{c}(\mathcal{B})$, which again requires the expensive complementation of $\mathcal{B}$. 
The emptiness problem for $\mathrm{ABW}$ is to decide, given an $\mathrm{ABW} \mathcal{A}$, whether $\mathcal{L}(\mathcal{A})=\emptyset$. This problem is also PSPACE-complete and it can be solved using a translation from ABW to NBW that preserves the language of the automaton [MH84. Again, this construction involves an exponential blow-up that makes explicit implementations feasible only for automata limited to around ten states. However, the emptiness problem for ABW is very important in practice for LTL model-checking as there exist efficient polynomial translations from LTL formulas to ABW GO01]. The classical construction is presented below.

Kupferman-Vardi construction. Complementation of ABW is straightforward by dualizing the transition function (by swapping $\wedge$ and $\vee$, and swapping true and false in each formulas) and interpreting the accepting condition $\alpha$ as a co-Büchi condition, i.e. a run $T_{w}$ is accepted if all its infinite paths have a suffix that contains no $\alpha$-nodes.

The result is an alternating co-Büchi automaton (ACW). The accepting runs of ACW have a layered structure that has been studied in KV01, where the notion of rank is defined. The rank is a nonnegative integer associated to each node of an accepting run $T_{w}$ of an ACW on a word $w$. Let $G_{0}=T_{w}$. Nodes of rank 0 are those nodes from which only finitely many nodes are reachable in $G_{0}$. Let $G_{1}$ be the run $T_{w}$ from which all nodes of rank 0 have been removed. Then, nodes of rank 1 are those nodes of $G_{1}$ from which no $\alpha$-node is reachable in $G_{1}$. For all $i \geq 2$, let $G_{i}$ be the run $T_{w}$ from which all nodes of rank $0, \ldots, i-1$ have been removed. Then, nodes of rank $2 i$ are those nodes of $G_{2 i}$ from which only finitely many nodes are reachable in $G_{2 i}$, and nodes of rank $2 i+1$ are those nodes of $G_{2 i+1}$ from which no $\alpha$-node is reachable in $G_{2 i+1}$. Intuitively, the rank of a node $(\ell, i)$ hints how difficult it is to prove that all the paths of $T_{w}$ that start in $(\ell, i)$ visit $\alpha$-nodes only finitely many times. It can be shown that every node has a rank between 0 and $2(\mid$ Loc $|-| \alpha \mid)$, and all $\alpha$-nodes have an even rank GKSV03. The layered structure of the runs of ACW induces a construction to complement ABW [KV01]. We present this construction directly for NBW.

Definition 2.3 ([KV01]). Given a NBW $\mathcal{A}=\langle$ Loc $, \iota, \Sigma, \delta, \alpha\rangle$ and an even number $k \in \mathbb{N}$, let $\operatorname{KV}(\mathcal{A}, k)=\left\langle\operatorname{Loc}^{\prime}, \iota^{\prime}, \Sigma, \delta^{\prime}, \alpha^{\prime}\right\rangle$ be an $\mathrm{ABW}$ such that:

- Loc $^{\prime}=\operatorname{Loc} \times[k]$ where $[k]=\{0,1, \ldots, k\}$. Intuitively, the automaton $\operatorname{KV}(\mathcal{A}, k)$ is in state $(\ell, n)$ after the first $i$ letters of the input word $w$ have been read if it guesses that the rank of the node $(\ell, i)$ in a run of $\mathcal{A}$ on $w$ is at most $n$;

- $\iota^{\prime}=(\iota, k)$;

- $\delta^{\prime}((\ell, i), \sigma)= \begin{cases}\text { false } & \text { if } \ell \in \alpha \text { and } i \text { is odd } \\ \bigwedge_{\ell^{\prime} \in \delta(\ell, \sigma)} \bigvee_{0 \leq i^{\prime} \leq i}\left(\ell^{\prime}, i^{\prime}\right) & \text { otherwise }\end{cases}$

For example, if $\delta(\ell, \sigma)=\left\{\ell_{1}, \ell_{2}\right\}$, then

$$
\delta^{\prime}((\ell, 2), \sigma)=\left(\left(\ell_{1}, 2\right) \vee\left(\ell_{1}, 1\right) \vee\left(\ell_{1}, 0\right)\right) \wedge\left(\left(\ell_{2}, 2\right) \vee\left(\ell_{2}, 1\right) \vee\left(\ell_{2}, 0\right)\right)
$$

- $\alpha^{\prime}=$ Loc $\times[k]^{\text {odd }}$ where $[k]^{\text {odd }}$ is the set of odd numbers in $[k]$.

The ABW specified by the Kupferman-Vardi construction accepts the complement language of $\mathcal{L}(\mathcal{A})$ and its size is quadratic in the size of the original automaton $\mathcal{A}$.

Theorem 2.4 ([KV01]). For all $N B W \mathcal{A}=\langle$ Loc, $\iota, \Sigma, \delta, \alpha\rangle$, for all $0 \leq k^{\prime} \leq k$, we have $\mathcal{L}\left(\operatorname{KV}\left(\mathcal{A}, k^{\prime}\right)\right) \subseteq \mathcal{L}(\operatorname{KV}(\mathcal{A}, k))$ and for $k=2(|\operatorname{Loc}|-|\alpha|)$, we have $\mathcal{L}(\operatorname{KV}(\mathcal{A}, k))=\mathcal{L}^{c}(\mathcal{A})$. 
Miyano-Hayashi construction. Classically, to check emptiness of ABW, a variant of the subset construction is applied that transforms the ABW into a NBW that accepts the same language [MH84]. Intuitively, the NBW maintains a set $s$ of states of the ABW that corresponds to a whole level of a guessed run DAG of the ABW. In addition, the NBW maintains a set $o$ of states that "owe" a visit to an accepting state. Whenever the set $o$ gets empty, meaning that every path of the guessed run has visited at least one accepting state, the set $o$ is initiated with the current level of the guessed run. It is asked that $o$ gets empty infinitely often in order to ensure that every path of the run DAG visits accepting states infinitely often. The construction is as follows.

Definition 2.5 (MH84]). Given an $\mathrm{ABW} \mathcal{A}=\langle$ Loc, $\iota, \Sigma, \delta, \alpha\rangle$, define $\mathrm{MH}(\mathcal{A})$ as the $\mathrm{NBW}$ $\left\langle 2^{\text {Loc }} \times 2^{\text {Loc }},(\{\iota\}, \emptyset), \Sigma, \delta^{\prime}, \alpha^{\prime}\right\rangle$ where $\alpha^{\prime}=2^{\text {Loc }} \times\{\emptyset\}$ and $\delta^{\prime}$ is defined, for all $\langle s, o\rangle \in 2^{\text {Loc }} \times 2^{\text {Loc }}$ and $\sigma \in \Sigma$, as follows:

- If $o \neq \emptyset$, then

$$
\delta^{\prime}(\langle s, o\rangle, \sigma)=\left\{\left\langle s^{\prime}, o^{\prime} \backslash \alpha\right\rangle \mid o^{\prime} \subseteq s^{\prime}, s^{\prime} \models \bigwedge_{\ell \in s} \delta(\ell, \sigma) \text { and } o^{\prime} \models \bigwedge_{\ell \in o} \delta(\ell, \sigma)\right\}
$$

- If $o=\emptyset$, then $\delta^{\prime}(\langle s, o\rangle, \sigma)=\left\{\left\langle s^{\prime}, s^{\prime} \backslash \alpha\right\rangle \mid s^{\prime} \models \bigwedge_{\ell \in s} \delta(\ell, \sigma)\right\}$.

The size of the Miyano-Hayashi construction is exponential in the size of the original automaton.

Theorem 2.6 ([MH84]). For all $A B W \mathcal{A}$, we have $\mathcal{L}(\mathrm{MH}(\mathcal{A}))=\mathcal{L}(\mathcal{A})$.

The size of the automaton obtained after the Kupferman-Vardi and the Miyano-Hayashi construction is an obstacle to the direct implementation of the method.

Direct complementation. In our solution, we implicitly use the two constructions to complement Büchi automata but, as we will see, we do not construct the automata. For the sake of clarity, we give below the specification of the automaton that would result from the composition of the two constructions. In the definition of the state space, we omit the states $(\ell, i)$ for $\ell \in \alpha$ and $i$ odd, as those states have no successor in the Kupferman-Vardi construction.

Definition 2.7. Given a NBW $\mathcal{A}=\langle$ Loc, $\iota, \Sigma, \delta, \alpha\rangle$ and an even number $k \in \mathbb{N}$, let $\operatorname{KVMH}(\mathcal{A}, k)=\left\langle Q_{k} \times Q_{k}, q_{\iota}, \Sigma, \delta^{\prime}, \alpha^{\prime}\right\rangle$ be a NBW such that:

- $Q_{k}=2^{(\operatorname{Loc} \times[k]) \backslash\left(\alpha \times \mathbb{N}^{o d d}\right)}$ where $\mathbb{N}^{\text {odd }}$ is the set of odd natural numbers;

- $q_{\iota}=(\{(\iota, k)\}, \emptyset)$;

- Let odd $=\operatorname{Loc} \times[k]^{o d d} ; \delta^{\prime}$ is defined for all $s, o \in Q_{k}$ and $\sigma \in \Sigma$, as follows:

- If $o \neq \emptyset$, then $\delta^{\prime}(\langle s, o\rangle, \sigma)$ is the set of pairs $\left\langle s^{\prime}, o^{\prime} \backslash\right.$ odd $\rangle$ such that:

(i) $o^{\prime} \subseteq s^{\prime}$

(ii) $\forall(\ell, n) \in s \cdot \forall \ell^{\prime} \in \delta(\ell, \sigma) \cdot \exists n^{\prime} \leq n:\left(\ell^{\prime}, n^{\prime}\right) \in s^{\prime}$;

(iii) $\forall(\ell, n) \in o \cdot \forall \ell^{\prime} \in \delta(\ell, \sigma) \cdot \exists n^{\prime} \leq n:\left(\ell^{\prime}, n^{\prime}\right) \in o^{\prime}$.

- If $o=\emptyset$, then $\delta^{\prime}(\langle s, o\rangle, \sigma)$ is the set of pairs $\left\langle s^{\prime}, s^{\prime} \backslash\right.$ odd $\rangle$ such that:

$\forall(\ell, n) \in s \cdot \forall \ell^{\prime} \in \delta(\ell, \sigma) \cdot \exists n^{\prime} \leq n:\left(\ell^{\prime}, n^{\prime}\right) \in s^{\prime}$.

- $\alpha^{\prime}=Q_{k} \times\{\emptyset\}$

We write $\langle s, o\rangle \stackrel{\sigma}{\rightarrow}_{\delta^{\prime}}\left\langle s^{\prime}, o^{\prime}\right\rangle$ to denote $\left\langle s^{\prime}, o^{\prime}\right\rangle \in \delta^{\prime}(\langle s, o\rangle, \sigma)$. 


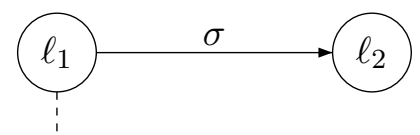

If $\curlyvee$

then
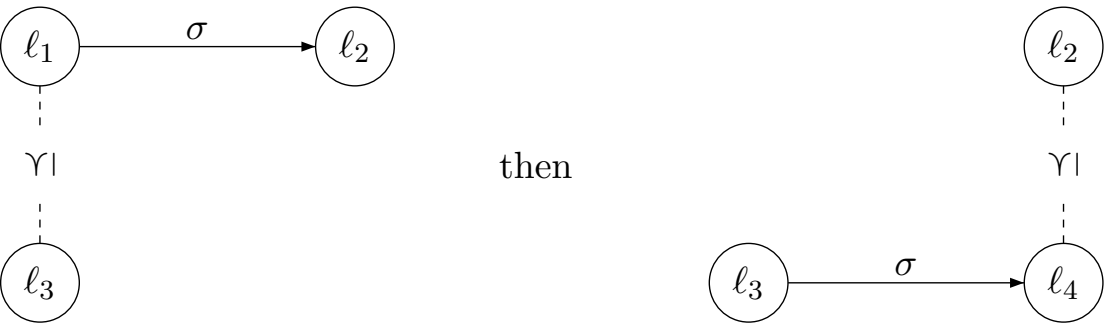

Figure 1: Simulation (Definition 3.1).

Theorem 2.8 ([KV01, MH84]). For every $N B W \mathcal{A}=\langle$ Loc, $\iota, \Sigma, \delta, \alpha\rangle$ and for all $0 \leq k^{\prime} \leq k$, we have $\mathcal{L}\left(\operatorname{KVMH}\left(\mathcal{A}, k^{\prime}\right)\right) \subseteq \mathcal{L}(\operatorname{KVMH}(\mathcal{A}, k))$. In case of $k=2(|\mathrm{Loc}|-|\alpha|)$, we also have $\mathcal{L}(\operatorname{KVMH}(\mathcal{A}, k))=\mathcal{L}^{c}(\mathcal{A})$.

In the sequel, we denote by $\operatorname{KVMH}(\mathcal{A})$ the automaton $\operatorname{KVMH}(\mathcal{A}, 2(|\operatorname{Loc}|-|\alpha|))$, and we denote by $Q \times Q$ its set of states (we omit the subscript $k$ ).

\section{Simulation Pre-Orders and Fixed Points}

Let $\mathcal{A}=\langle$ Loc $, \iota, \Sigma, \delta, \alpha\rangle$ be a NBW. Let $\left\langle 2^{\text {Loc }}, \subseteq, \cup, \cap, \emptyset\right.$, Loc $\rangle$ be the powerset lattice of locations. The fixed point formula $\mathcal{F}_{\mathcal{A}} \equiv \nu y \cdot \mu x \cdot\left(\operatorname{Pre}^{\mathcal{A}}(x) \cup\left(\operatorname{Pre}^{\mathcal{A}}(y) \cap \alpha\right)\right)$ can be used to check emptiness of $\mathcal{A}$ as we have $\mathcal{L}(\mathcal{A}) \neq \emptyset$ iff $\iota \in \mathcal{F}_{\mathcal{A}}$. Intuitively, the greatest fixed point $\nu y$ in $\mathcal{F}_{\mathcal{A}}$ computes in the $n$-th iteration the set of states from which $n$ accepting states can be visited with some word. When this set stabilizes, infinitely many visits to an accepting state are possible.

We show in this section that a certain structural property of the NBW is tightly correlated to the structure of the sets that are computed by the fixed point $\mathcal{F}_{\mathcal{A}}$. The key property is the notion of simulation relation for finite automata. Let $\preceq \subseteq$ Loc $\times$ Loc be a pre-order and let $\ell_{1} \prec \ell_{2}$ iff $\ell_{1} \preceq \ell_{2}$ and $\ell_{2} \npreceq \ell_{1}$.

Definition 3.1. A pre-order $\preceq$ is a simulation for $\mathcal{A}$ iff the following properties hold:

- for all $\ell_{1}, \ell_{2}, \ell_{3} \in$ Loc, for all $\sigma \in \Sigma$, if $\ell_{3} \preceq \ell_{1}$ and $\ell_{2} \in \delta\left(\ell_{1}, \sigma\right)$ then there exists $\ell_{4} \in$ Loc such that $\ell_{4} \preceq \ell_{2}$ and $\ell_{4} \in \delta\left(\ell_{3}, \sigma\right)$ (see illustration in Figure 1);

- for all $\ell \in \alpha$, for all $\ell^{\prime} \in$ Loc, if $\ell^{\prime} \preceq \ell$ then $\ell^{\prime} \in \alpha$.

Downward-closed sets. A set $L \subseteq$ Loc is $\preceq$-closed iff for all $\ell_{1}, \ell_{2} \in$ Loc, if $\ell_{1} \preceq \ell_{2}$ and $\ell_{2} \in L$ then $\ell_{1} \in L$. The $\preceq$-closure of $L$, is the set $\downarrow L=\left\{\ell \in \operatorname{Loc} \mid \exists \ell^{\prime} \in L: \ell \preceq \ell^{\prime}\right\}$. We denote by $\operatorname{Max}(L)$ the set of $\preceq$-maximal elements of $L: \operatorname{Max}(L)=\left\{\ell \in L \mid \nexists \ell^{\prime} \in L: \ell \prec \ell^{\prime}\right\}$. For any $\preceq$-closed set $L \subseteq$ Loc, we have $L=\downarrow \operatorname{Max}(L)$. Furthermore, if $\preceq$ is a partial order, then $\operatorname{Max}(L)$ is an antichain of elements and it can serve as a canonical representation of $L$.

Our goal is to show that the operators involved in the fixed point formula $\mathcal{F}_{\mathcal{A}}$ preserve $\preceq$-closedness. This is true for union and intersection, for all relations $\preceq$.

Lemma 3.2. For all relations $\preceq$, for all $\preceq$-closed sets $L_{1}, L_{2}$, the sets $L_{1} \cup L_{2}$ and $L_{1} \cap L_{2}$ are $\preceq$-closed. 
The next lemma shows that simulation relations are necessary (and also sufficient) to guarantee preservation of $\preceq$-closedness under the Pre operator. Note that many other notions of simulation pre-orders have been defined for Büchi automata, see [EWS05] 1 ]

Lemma 3.3. Let $\mathcal{A}=\langle$ Loc, $\iota, \Sigma, \delta, \alpha\rangle$ be a $N B W$. A pre-order $\preceq \subseteq$ Loc $\times$ Loc is a simulation for $\mathcal{A}$ if and only if the following two properties hold:

(a) the set $\alpha$ is $\preceq$-closed.

(b) for all $\preceq$-closed sets $L \subseteq$ Loc, for all $\sigma \in \Sigma$, $\operatorname{Pre}_{\sigma}^{\mathcal{A}}(L)$ is $\preceq$-closed;

Proof. First, assume that $\preceq$ is a simulation for $\mathcal{A}$. Then, the set $\alpha$ is $\preceq$-closed by Definition 3.1, which establishes (a). To prove (b), let $L \subseteq$ Loc be a $\preceq$-closed set and let $\sigma \in \Sigma$. For all $\ell_{1} \in \operatorname{Pre}_{\sigma}^{\mathcal{A}}(L)$ there exists $\ell_{2} \in L$ such that $\ell_{2} \in \delta\left(\ell_{1}, \sigma\right)$. By Definition 3.1, for all $\ell_{3} \preceq \ell_{1}$ there exists $\ell_{4} \in$ Loc such that $\ell_{4} \preceq \ell_{2}$ and $\ell_{4} \in \delta\left(\ell_{3}, \sigma\right)$ (see Figure 11). So $\ell_{4} \in L$ since $L$ is $\preceq$-closed and $\ell_{2} \in L$, and thus $\ell_{3} \in \operatorname{Pre}_{\sigma}^{\mathcal{A}}(L)$ which shows that $\operatorname{Pre}_{\sigma}^{\mathcal{A}}(L)$ is $\preceq$-closed.

Second, assume that (a) and (b) hold, and show that $\preceq$ satisfies Definition 3.1. By (a), for all $\ell \in \alpha$ and for all $\ell^{\prime} \preceq \ell$, we have $\ell^{\prime} \in \alpha$. Now, let $\ell_{1}, \ell_{2}, \ell_{3} \in$ Loc and $\sigma \in \Sigma$ such that $\ell_{3} \preceq \ell_{1}$ and $\ell_{2} \in \delta\left(\ell_{1}, \sigma\right)$. Consider the $\preceq$-closed set $L_{2}=\downarrow\left\{\ell_{2}\right\}$. By (b), the set $\operatorname{Pre}_{\sigma}^{\mathcal{A}}\left(L_{2}\right)$ is $\preceq$-closed and thus $\ell_{3} \in \operatorname{Pre}_{\sigma}^{\mathcal{A}}\left(L_{2}\right)$. Therefore, there exists $\ell_{4} \in L_{2}$ (i.e. $\ell_{4} \preceq \ell_{2}$ ) such that $\ell_{4} \in \delta\left(\ell_{3}, \sigma\right)$. Hence, $\preceq$ is a simulation for $\mathcal{A}$.

Lemmas 3.2 and 3.3 entail that all sets computed in the iterations of the fixed point formula $\mathcal{F}_{\mathcal{A}}$ are $\preceq$-closed for any simulation $\preceq$ for $\mathcal{A}$. We can take advantage of this fact to use a compact representation of those sets, namely their maximal elements. This would indeed reduce the size of the sets to manipulate by the fixed point algorithms (possibly exponentially as we will see later). Notice that in general, this compact representation can make more difficult the computation of the Pre operator. To illustrate this, consider the example in Figure 2 where we want to compute $\operatorname{Pre}_{\sigma}(\downarrow\{\ell\})$. More precisely, given $\ell$ we need to compute the maximal elements of the $\preceq$-closed set $\operatorname{Pre}_{\sigma}(\downarrow\{\ell\})$. The set $\downarrow\{\ell\}$ is delimited by the dashed curve in the figure. First, note that applying $\operatorname{Pre}_{\sigma}$ to $\{\ell\}$ would give the empty set from which the correct result can obviously not be extracted. Second, if we assume that the states $\ell_{1}, \ldots, \ell_{k}$ are $\preceq$-incomparable, then the result is $\operatorname{Max}\left(\operatorname{Pre}_{\sigma}(\downarrow\{\ell\})\right)=\left\{\ell_{1}, \ldots, \ell_{k}\right\}$, which shows that essentially any set can be obtained, including sets of maximal elements that are huge or difficult to manipulate symbolically. Third, even if the result is compact (e.g., if $\ell_{i} \preceq \ell_{1}$ for all $1 \leq i \leq k$, then the result is the singleton $\left\{\ell_{1}\right\}$ ), the computation may somehow require to enumerate all the $\ell_{i}$ for $i=1,2, \ldots, k$ where $k$ may be for instance exponential in the size of the problem.

The above remarks show that for each particular application (i.e., for each class of automata, and each particular simulation $\preceq$ that we use), we need (1) to define a predecessor operator $\operatorname{Pre}^{\text {abs }}$ that applies to maximal elements, such that $\operatorname{Pre}^{\text {abs }}(\operatorname{Max}(L))=\operatorname{Max}(\operatorname{Pre}(L))$ for all $\preceq$-closed sets $L,(2)$ to present an algorithm to compute this operator, and establish its correctness, and (3) to study the complexity of such an algorithm.

Finally, note that the way to compute $\operatorname{Max}\left(L_{1} \cap L_{2}\right)$ given $\operatorname{Max}\left(L_{1}\right)$ and $\operatorname{Max}\left(L_{2}\right)$ should also be defined for each application, while for union, the following general rule applies: $\operatorname{Max}\left(L_{1} \cup L_{2}\right)=\operatorname{Max}\left(\operatorname{Max}\left(L_{1}\right) \cup \operatorname{Max}\left(L_{2}\right)\right)$.

In the next sections, we show that the NBW that we have to analyze in the automatabased approach to model-checking are all equipped with a simulation pre-order that can be

\footnotetext{
${ }^{1}$ In [EWS05], the simulation of Definition 3.1] is called direct simulation.
} 


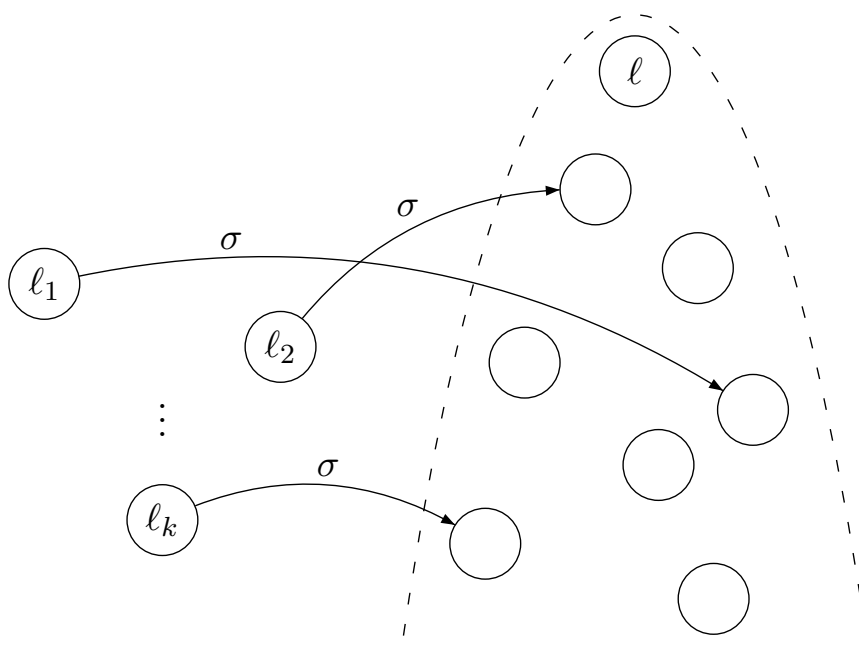

Figure 2: Computing the predecessors of a $\preceq$-closed set.

exploited to compute efficiently the intersection and the predecessor operators. Hence, we show that the expected efficiency in terms of space consumption of the antichain representation does not come at the price of a blow-up in the computation times of these operators. We do so for the emptiness problem of ABW, and for the universality and language inclusion problems for NBW. All these problems can be reduced to the emptiness problem of NBW that are obtained by specific constructions (analogous of the powerset construction), for which simulation relations need not to be computed for each instance of the problems, but can be defined generically (like set inclusion is such a relation for the classical powerset construction).

\section{EMptiness of ABW}

We now show how to apply Lemmas 3.2 and 3.3 to check more efficiently the emptiness of ABW. Let $\mathcal{A}_{1}=\left\langle\operatorname{Loc}_{1}, \iota_{1}, \Sigma, \delta_{1}, \alpha_{1}\right\rangle$ be an ABW for which we want to decide whether $\mathcal{L}\left(\mathcal{A}_{1}\right)=\emptyset$. We know that the (exponential) Miyano-Hayashi construction gives a NBW $\mathcal{A}_{2}=\operatorname{MH}\left(\mathcal{A}_{1}\right)$ such that $\mathcal{L}\left(\mathcal{A}_{2}\right)=\mathcal{L}\left(\mathcal{A}_{1}\right)$. The emptiness of $\mathcal{A}_{1}$ (or equivalently of $\mathcal{A}_{2}$ ) can be decided more efficiently by computing the fixed point $\mathcal{F}_{\mathcal{A}_{2}}$ and without constructing explicitly $\mathcal{A}_{2}$. To do so, we establish the existence of a simulation for $\mathcal{A}_{2}$ for which we can compute $\cup, \cap$ and Pre by manipulating only maximal elements of closed sets of locations.

Definition 4.1. Let $\mathrm{MH}\left(\mathcal{A}_{1}\right)=\left\langle\operatorname{Loc}_{2}, \iota_{2}, \Sigma, \delta_{2}, \alpha_{2}\right\rangle$. Remember that $\operatorname{Loc}_{2} \subseteq 2^{\text {Loc }_{1}} \times 2^{\text {Loc }_{1}}$. Define the pre-order $\preceq_{\text {alt }} \subseteq \operatorname{Loc}_{2} \times \operatorname{Loc}_{2}$ such that $\langle s, o\rangle \preceq_{\text {alt }}\left\langle s^{\prime}, o^{\prime}\right\rangle$ iff $(i) s \subseteq s^{\prime},(i i) o \subseteq o^{\prime}$, and $($ iii $) o=\emptyset$ iff $o^{\prime}=\emptyset$.

Note that the pre-order $\preceq_{\text {alt }}$ is a partial order. As a consequence, given a set of pairs $L=\left\{\left\langle s_{1}, o_{1}\right\rangle,\left\langle s_{2}, o_{2}\right\rangle, \ldots,\left\langle s_{n}, o_{n}\right\rangle\right\}$, the set $\operatorname{Max}(L)$ is an antichain and identifies $L$.

Lemma 4.2. For all $A B W \mathcal{A}_{1}$, the partial order $\preceq_{\text {alt }}$ is a simulation for $\operatorname{MH}\left(\mathcal{A}_{1}\right)$.

Proof. Let $\mathcal{A}_{1}=\left\langle\operatorname{Loc}_{1}, \iota_{1}, \Sigma, \delta_{1}, \alpha_{1}\right\rangle$ and $\mathrm{MH}\left(\mathcal{A}_{1}\right)=\left\langle\operatorname{Loc}_{2}, \iota_{2}, \Sigma, \delta_{2}, \alpha_{2}\right\rangle$. First, let $\sigma \in \Sigma$ and $\left\langle s_{1}, o_{1}\right\rangle,\left\langle s_{2}, o_{2}\right\rangle,\left\langle s_{3}, o_{3}\right\rangle \in \operatorname{Loc}_{2}$ be such that $\left\langle s_{1}, o_{1}\right\rangle \stackrel{\sigma}{\rightarrow} \delta_{2}\left\langle s_{2}, o_{2}\right\rangle$ and $\left\langle s_{3}, o_{3}\right\rangle \preceq$ alt 


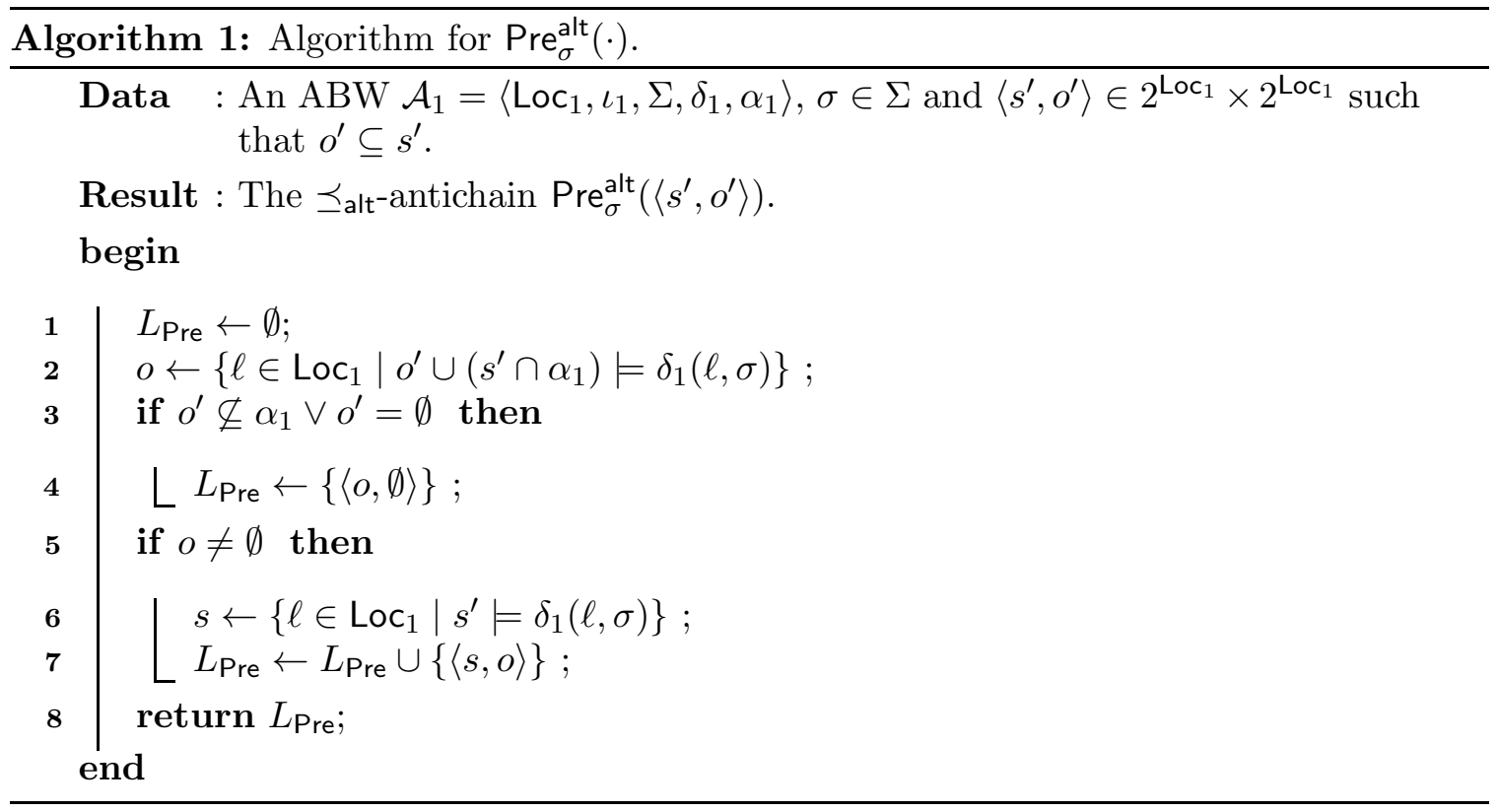

$\left\langle s_{1}, o_{1}\right\rangle$. We show that there exists $\left\langle s_{4}, o_{4}\right\rangle \in \operatorname{Loc}_{2}$ such that $\left\langle s_{3}, o_{3}\right\rangle \stackrel{\sigma}{\rightarrow}_{\delta_{2}}\left\langle s_{4}, o_{4}\right\rangle$ and $\left\langle s_{4}, o_{4}\right\rangle \preceq$ alt $\left\langle s_{2}, o_{2}\right\rangle$. Let us consider the case where $o_{1}=\emptyset$. Then we have $o_{3}=\emptyset$ by definition of $\preceq_{\text {alt }}$ and $\delta_{2}\left(\left\langle s_{1}, o_{1}\right\rangle, \sigma\right)=\left\{\left\langle s^{\prime}, s^{\prime} \backslash \alpha_{1}\right\rangle \mid s^{\prime} \models \bigwedge_{l \in s_{1}} \delta_{1}(l, \sigma)\right\}$, this set being contained in $\delta_{2}\left(\left\langle s_{3}, o_{3}\right\rangle, \sigma\right)=\left\{\left\langle s^{\prime}, s^{\prime} \backslash \alpha_{1}\right\rangle \mid s^{\prime} \models \bigwedge_{l \in s_{3}} \delta_{1}(l, \sigma)\right\}$ as $s_{3}$ puts less constraints than $s_{1}$ since $s_{3} \subseteq s_{1}$. A similar reasoning holds if $o_{1} \neq \emptyset$. Second, let $\left\langle s_{1}, o_{1}\right\rangle \in \alpha_{2}$ and let $\left\langle s_{2}, o_{2}\right\rangle \preceq_{\text {alt }}\left\langle s_{1}, o_{1}\right\rangle$. By definition of $\alpha_{2}$, we know that $o_{1}=\emptyset$, and by definition of $\preceq_{\text {alt }}$ we have $o_{2}=\emptyset$ and so $\left\langle s_{2}, o_{2}\right\rangle \in \alpha_{2}$.

According to Lemmas 3.2 and 3.3 , all the sets that we compute to evaluate $\mathcal{F}_{\mathcal{A}_{2}}$ are

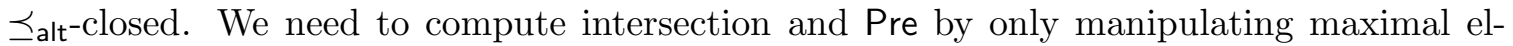
ements. Given $\left\langle s_{1}, o_{1}\right\rangle,\left\langle s_{2}, o_{2}\right\rangle$, we take $\langle s, o\rangle$ such that $\downarrow\langle s, o\rangle=\downarrow\left\langle s_{1}, o_{1}\right\rangle \cap \downarrow\left\langle s_{2}, o_{2}\right\rangle$ as follows:

$$
\langle s, o\rangle= \begin{cases}\left\langle s_{1} \cap s_{2}, o_{1} \cap o_{2}\right\rangle & \text { if } o_{1} \cap o_{2} \neq \emptyset, \\ \left\langle s_{1} \cap s_{2}, \emptyset\right\rangle & \text { if } o_{1}=o_{2}=\emptyset,\end{cases}
$$

and otherwise the intersection is empty.

Algorithm 1 computes the maximal elements of the set of $\sigma$-predecessors of the $\preceq$ alt-closure of a pair $\left\langle s^{\prime}, o^{\prime}\right\rangle$. This allows to compute the maximal elements of the set of predecessors of any $\preceq_{\text {alt }}$-closed set by just manipulating its maximal elements, since $\operatorname{Pre}^{\mathcal{A}}\left(L_{1} \cup L_{2}\right)=$ $\bigcup_{\sigma \in \Sigma} \operatorname{Pre}_{\sigma}^{\mathcal{A}}\left(L_{1}\right) \cup \operatorname{Pre}_{\sigma}^{\mathcal{A}}\left(L_{2}\right)$.

Note that our algorithm runs in polynomial time, more precisely in $O\left(\left|\operatorname{Loc}_{1}\right| \cdot\left\|\delta_{1}\right\|\right)$ where $\left\|\delta_{1}\right\|$ is the size of the transition relation, defined as the maximal number of boolean connectives in a formula $\delta_{1}(\ell, \sigma)$.

Theorem 4.3. Given an $A B W \mathcal{A}_{1}=\left\langle\operatorname{Loc}_{1}, \iota_{1}, \Sigma, \delta_{1}, \alpha_{1}\right\rangle, \sigma \in \Sigma$ and $\left\langle s^{\prime}, o^{\prime}\right\rangle \in 2^{\text {Loc }_{1}} \times 2^{\text {Loc }_{1}}$ such that $o^{\prime} \subseteq s^{\prime}$, the set $L_{\mathrm{Pre}}=\operatorname{Pre}_{\sigma}^{\text {alt }}(\langle s, o\rangle)$ computed by Algorithm 1 is an $\preceq_{\text {alt }}$-antichain such that $\downarrow L_{\text {Pre }}=\operatorname{Pre}_{\sigma}^{\mathcal{A}_{2}}\left(\downarrow\left\{\left\langle s^{\prime}, o^{\prime}\right\rangle\right\}\right)$ where $\mathcal{A}_{2}=\operatorname{MH}\left(\mathcal{A}_{1}\right)$.

Proof. Let $\mathcal{A}_{2}=\operatorname{MH}\left(\mathcal{A}_{1}\right)=\left\langle\operatorname{Loc}_{2}, \iota_{2}, \Sigma, \delta_{2}, \alpha_{2}\right\rangle$. The following entails that $\downarrow L L_{\text {Pre }}=\operatorname{Pre}_{\sigma}^{\mathcal{A}_{2}}(\downarrow$ $\left.\left\{\left\langle s^{\prime}, o^{\prime}\right\rangle\right\}\right)$ : 
(a) $L_{\text {Pre }} \subseteq \operatorname{Pre}_{\sigma}^{\mathcal{A}_{2}}\left(\downarrow\left\{\left\langle s^{\prime}, o^{\prime}\right\rangle\right\}\right)$, and

(b) for all $\left\langle s_{1}, o_{1}\right\rangle \in \operatorname{Pre}_{\sigma}^{\mathcal{A}_{2}}\left(\downarrow\left\{\left\langle s^{\prime}, o^{\prime}\right\rangle\right\}\right)$,

there exists $\langle s, o\rangle \in L_{\text {Pre }}$ such that $\left\langle s_{1}, o_{1}\right\rangle \preceq$ alt $\langle s, o\rangle$.

To prove (a), we first show that $\langle s, o\rangle \stackrel{\sigma}{\rightarrow} \delta_{2}\left\langle s^{\prime}, o^{\prime}\right\rangle$ where $\langle s, o\rangle$ is added to $L_{\text {Pre }}$ at line 7 of Algorithm 1. By the test of line 5, we have $o \neq \emptyset$. According to Definition 2.5 of $\mathrm{MH}(\cdot)$, we check that there exists a set $o^{\prime \prime} \subseteq s^{\prime}$ such that $o^{\prime}=o^{\prime \prime} \backslash \alpha_{1}$ (we take $o^{\prime \prime}=o^{\prime} \cup\left(s^{\prime} \cap \alpha_{1}\right)$ ), and the following conditions hold:

(i) $s^{\prime} \models \bigwedge_{\ell \in s} \delta_{1}(\ell, \sigma)$ since we have $s^{\prime} \models \delta_{1}(\ell, \sigma)$ for all $\ell \in s$ by line 6 of Alg. 1 .

(ii) $o^{\prime \prime} \models \bigwedge_{\ell \in o} \delta_{1}(\ell, \sigma)$ since we have $o^{\prime \prime} \models \delta_{1}(\ell, \sigma)$ for all $\ell \in o$ by line 2 of Alg. 1 .

Second, we show that $\langle o, \emptyset\rangle \stackrel{\sigma}{\rightarrow}_{\delta_{2}}\left\langle s^{\prime \prime}, o^{\prime \prime}\right\rangle$ for some $\left\langle s^{\prime \prime}, o^{\prime \prime}\right\rangle \preceq_{\text {alt }}\left\langle s^{\prime}, o^{\prime}\right\rangle$ where $\langle o, \emptyset\rangle$ is added to $L_{\text {Pre }}$ at line 4 of Algorithm 1. We take $s^{\prime \prime}=o^{\prime} \cup\left(s^{\prime} \cap \alpha_{1}\right)$ and $o^{\prime \prime}=s^{\prime \prime} \backslash \alpha_{1}$. Since $o^{\prime} \subseteq s^{\prime}$, we have $(a) s^{\prime \prime} \subseteq s^{\prime}$, and we have $(b) o^{\prime \prime}=o^{\prime} \backslash \alpha_{1} \subseteq o^{\prime}$. Let us establish that $(c)$ $o^{\prime}=\emptyset$ iff $o^{\prime \prime}=\emptyset$. If $o^{\prime}=\emptyset$ then $o^{\prime \prime}=\emptyset$ since $o^{\prime \prime} \subseteq o^{\prime}$. If $o^{\prime} \neq \emptyset$ then by the test of line 3, we have $o^{\prime} \nsubseteq \alpha_{1}$ and thus $o^{\prime \prime}=o^{\prime} \backslash \alpha_{1} \neq \emptyset$. Hence we have $\left\langle s^{\prime \prime}, o^{\prime \prime}\right\rangle \preceq$ alt $\left\langle s^{\prime}, o^{\prime}\right\rangle$, and by line 2 of the algorithm, we have $s^{\prime \prime} \models \delta_{1}(\ell, \sigma)$ for all $\ell \in o$, and thus $s^{\prime \prime} \models \bigwedge_{\ell \in o} \delta_{1}(\ell, \sigma)$. Therefore $\langle o, \emptyset\rangle \stackrel{\sigma}{\rightarrow}_{\delta_{2}}\left\langle s^{\prime \prime}, o^{\prime \prime}\right\rangle$.

To prove (b), assume that there exist $\left\langle s_{1}, o_{1}\right\rangle$ and $\left\langle s_{1}^{\prime}, o_{1}^{\prime}\right\rangle$ such that $\left\langle s_{1}, o_{1}\right\rangle \stackrel{\sigma}{\rightarrow}_{\delta_{2}}\left\langle s_{1}^{\prime}, o_{1}^{\prime}\right\rangle$ and $\left\langle s_{1}^{\prime}, o_{1}^{\prime}\right\rangle \preceq_{\text {alt }}\left\langle s^{\prime}, o^{\prime}\right\rangle$. We have to show that there exists $\langle s, o\rangle \in L_{\text {Pre }}$ such that $\left\langle s_{1}, o_{1}\right\rangle \preceq$ alt $\langle s, o\rangle$.

First, assume that $o_{1} \neq \emptyset$. Since $\left\langle s_{1}, o_{1}\right\rangle \stackrel{\sigma}{\rightarrow}_{\delta_{2}}\left\langle s_{1}^{\prime}, o_{1}^{\prime}\right\rangle$, we have:

(i) for all $\ell \in s_{1}, s_{1}^{\prime} \models \delta_{1}(\ell, \sigma)$ and since $s_{1}^{\prime} \subseteq s^{\prime}$ also $s^{\prime} \models \delta_{1}(\ell, \sigma)$. Let $s$ be the set defined at line 6 of Algorithm 1. For all $\ell \in$ Loc, if $s^{\prime} \models \delta_{1}(\ell, \sigma)$ then $\ell \in s$. Hence, $s_{1} \subseteq s$.

(ii) for all $\ell \in o_{1}, o_{1}^{\prime \prime} \models \delta_{1}(\ell, \sigma)$ for some $o_{1}^{\prime \prime} \subseteq s_{1}^{\prime}$ such that $o_{1}^{\prime}=o_{1}^{\prime \prime} \backslash \alpha_{1}$. Hence necessarily $o_{1}^{\prime \prime} \subseteq o_{1}^{\prime} \cup\left(s_{1}^{\prime} \cap \alpha_{1}\right) \subseteq o^{\prime} \cup\left(s^{\prime} \cap \alpha_{1}\right)$ and thus for all $\ell \in o_{1}, o^{\prime} \cup\left(s^{\prime} \cap \alpha_{1}\right) \models \delta_{1}(\ell, \sigma)$. Let $o$ be the set defined at line 2 of Algorithm 1. For all $\ell \in$ Loc, if $o^{\prime} \cup\left(s^{\prime} \cap \alpha_{1}\right) \models \delta_{1}(\ell, \sigma)$ then $\ell \in o$. Hence, $o_{1} \subseteq o$ and $o \neq \emptyset$.

Hence, $\langle s, o\rangle$ which is added to $L_{\text {Pre }}$ by Alg. 1 at line 7 satisfies $\left\langle s_{1}, o_{1}\right\rangle \preceq$ alt $\langle s, o\rangle$.

Second, assume that $o_{1}=\emptyset$. Since $\left\langle s_{1}, o_{1}\right\rangle \stackrel{\sigma}{\rightarrow} \delta^{\prime}\left\langle s_{1}^{\prime}, o_{1}^{\prime}\right\rangle$ and $o_{1}=\emptyset$, we know that for all $\ell \in s_{1}, s_{1}^{\prime} \models \delta_{1}(\ell, \sigma)$ and $o_{1}^{\prime}=s_{1}^{\prime} \backslash \alpha_{1}$. Let $s^{\prime \prime}=o^{\prime} \cup\left(s^{\prime} \cap \alpha_{1}\right)$ so we have $(a)$ $s_{1}^{\prime} \cap \alpha_{1} \subseteq s^{\prime} \cap \alpha_{1} \subseteq s^{\prime \prime}$ and $(b) s_{1}^{\prime} \backslash \alpha_{1}=o_{1}^{\prime} \subseteq o^{\prime} \subseteq s^{\prime \prime}$. Hence, $s_{1}^{\prime} \subseteq s^{\prime \prime}$ and thus for all $\ell \in s_{1}, s^{\prime \prime} \models \delta_{1}(\ell, \sigma)$. Let $o$ be the set defined at line 2 of Algorithm 1. For all $\ell \in$ Loc, if $s^{\prime \prime} \models \delta_{1}(\ell, \sigma)$ then $\ell \in o$. Hence, $s_{1} \subseteq o$ and $\left\langle s_{1}, \emptyset\right\rangle \preceq_{\text {alt }}\langle o, \emptyset\rangle$ where $\langle o, \emptyset\rangle$ is added to $L_{\text {Pre }}$ by Algorithm 1 at line 4. Notice that the test at line 3 is satisfied because $o_{1}^{\prime}=s_{1}^{\prime} \backslash \alpha_{1}$ implies that $o_{1}^{\prime} \nsubseteq \alpha_{1} \vee o_{1}^{\prime}=\emptyset$ and since $\left\langle s_{1}^{\prime}, o_{1}^{\prime}\right\rangle \preceq_{\text {alt }}\left\langle s^{\prime}, o^{\prime}\right\rangle$, we have $o^{\prime} \nsubseteq \alpha_{1} \vee o^{\prime}=\emptyset$.

\section{UNIVERSALITY OF NBW}

We present a new algorithm to check universality of NBW, based the existence of a simple simulation relation for the complement automaton of NBW given by Definition 2.7.

Definition 5.1. Given an NBW $\mathcal{A}=\langle$ Loc, $\iota, \Sigma, \delta, \alpha\rangle$, let $\operatorname{KVMH}(\mathcal{A})=\left\langle Q \times Q, q_{\iota}, \Sigma, \delta^{\prime}, \alpha^{\prime}\right\rangle$. Define the pre-order $\preceq_{\text {univ }} \subseteq(Q \times Q) \times(Q \times Q)$ as follows: for all $s, s^{\prime}, o, o^{\prime} \in Q$, let $\langle s, o\rangle \preceq$ univ $\left\langle s^{\prime}, o^{\prime}\right\rangle$ iff the following conditions hold:

- for all $(\ell, n) \in s$, there exists $n^{\prime} \leq n$ such that $\left(\ell, n^{\prime}\right) \in s^{\prime}$;

- for all $(\ell, n) \in o$, there exists $n^{\prime} \leq n$ such that $\left(\ell, n^{\prime}\right) \in o^{\prime}$; 
- $o=\emptyset$ iff $o^{\prime}=\emptyset$.

This relation formalizes the intuition that it is easier to accept a word in $\operatorname{KVMH}(\mathcal{A})$ from a given location with a high rank than with a small rank. This is because the rank is always decreasing along every path of the runs of $\operatorname{KV}(\mathcal{A})$, and so a small rank is always simulated by a greater rank. Hence, essentially the minimal rank of each location of $s$ and $o$ is relevant to define the pre-order $\preceq$ univ. The third condition requires that only accepting states simulate accepting states.

Lemma 5.2. For all $N B W \mathcal{A}$, the pre-order $\preceq_{\text {univ }}$ is a simulation for the $N B W \operatorname{KVMH}(\mathcal{A})$.

Proof. Let $\mathcal{A}=\langle$ Loc, $\iota, \Sigma, \delta, \alpha\rangle$ and $\operatorname{KVMH}(\mathcal{A})=\left\langle Q \times Q, q_{\iota}, \Sigma, \delta^{\prime}, \alpha^{\prime}\right\rangle$. First, we show that for all $\left\langle s_{1}, o_{1}\right\rangle,\left\langle s_{2}, o_{2}\right\rangle,\left\langle s_{3}, o_{3}\right\rangle \in Q \times Q$, for all $\sigma \in \Sigma$, if $\left\langle s_{1}, o_{1}\right\rangle \stackrel{\sigma}{\rightarrow} \delta_{\delta^{\prime}}\left\langle s_{2}, o_{2}\right\rangle$ and $\left\langle s_{3}, o_{3}\right\rangle \preceq$ $\left\langle s_{1}, o_{1}\right\rangle$ then $\left\langle s_{3}, o_{3}\right\rangle \stackrel{\sigma}{\rightarrow}_{\delta^{\prime}}\left\langle s_{2}, o_{2}\right\rangle$. Notice that we have trivially $\left\langle s_{2}, o_{2}\right\rangle \preceq_{\text {univ }}\left\langle s_{2}, o_{2}\right\rangle$. We give the proof for $o_{1} \neq \emptyset$. The case $o_{1}=\emptyset$ is proven similarly. According to Definition 2.7, since $\left\langle s_{1}, o_{1}\right\rangle \stackrel{\sigma}{\rightarrow}_{\delta^{\prime}}\left\langle s_{2}, o_{2}\right\rangle$ we have

(i) $\forall\left(\ell, n_{1}\right) \in s_{1} \cdot \forall \ell^{\prime} \in \delta(\ell, \sigma) \cdot \exists n_{2} \leq n_{1}:\left(\ell^{\prime}, n_{2}\right) \in s_{2}$ and

(ii) $\forall\left(\ell, n_{1}\right) \in o_{1} \cdot \forall \ell^{\prime} \in \delta(\ell, \sigma) \cdot \exists n_{2} \leq n_{1}:\left(\ell^{\prime}, n_{2}\right) \in o_{2}$

Since $\left\langle s_{3}, o_{3}\right\rangle \preceq\left\langle s_{1}, o_{1}\right\rangle$, we have $o_{3} \neq \emptyset$ and

$\left(i^{\prime}\right) \quad \forall\left(\ell, n_{3}\right) \in s_{3} \cdot \exists n_{1} \leq n_{3}:\left(\ell, n_{1}\right) \in s_{1}$ and

(ii') $\forall\left(\ell, n_{3}\right) \in o_{3} \cdot \exists n_{1} \leq n_{3}:\left(\ell, n_{1}\right) \in o_{1}$

Combining $(i)$ and $\left(i^{\prime}\right)$ yields $\forall\left(\ell, n_{3}\right) \in s_{3} \cdot \forall \ell^{\prime} \in \delta(\ell, \sigma) \cdot \exists n_{2} \leq n_{3}:\left(\ell^{\prime}, n_{2}\right) \in s_{2}:$, and combining (ii) and $\left(i i^{\prime}\right)$ yields $\forall\left(\ell, n_{3}\right) \in o_{3} \cdot \forall \ell^{\prime} \in \delta(\ell, \sigma) \cdot \exists n_{2} \leq n_{3}:\left(\ell^{\prime}, n_{2}\right) \in o_{2}$. Since $o_{3} \neq \emptyset$, this implies that $\left\langle s_{3}, o_{3}\right\rangle \stackrel{\sigma}{\rightarrow} \delta^{\prime}\left\langle s_{2}, o_{2}\right\rangle$.

Second, for all $\langle s, o\rangle \in \alpha^{\prime}$ we have $o=\emptyset$, and thus for all $\left\langle s^{\prime}, o^{\prime}\right\rangle \in Q \times Q$, if $\left\langle s^{\prime}, o^{\prime}\right\rangle \preceq\langle s, o\rangle$ then $o^{\prime}=\emptyset$ so that $\left\langle s^{\prime}, o^{\prime}\right\rangle \in \alpha^{\prime}$.

Hence $\preceq_{\text {univ }}$ is a simulation for $\operatorname{KVMH}(\mathcal{A})$.

According to Lemmas 3.2 and 3.3, all intermediate sets that are computed by the fixed point $\mathcal{F}_{\mathcal{A}^{c}}$ to check emptiness of $\mathcal{A}^{c}=\operatorname{KVMH}(\mathcal{A})$ (and thus universality of $\mathcal{A}$ ) are

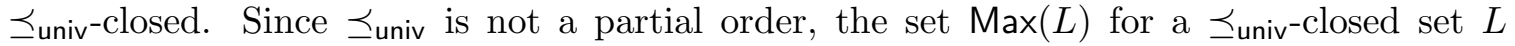

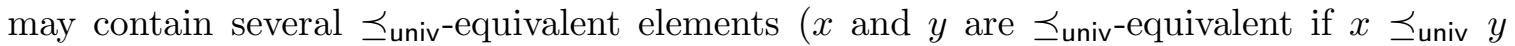
and $y \preceq$ univ $x)$. For example, the set $\left\{\left\langle\left\{(\ell, 3),\left(\ell^{\prime}, 4\right)\right\}, \emptyset\right\rangle\right\}$ is $\preceq_{\text {univ-equivalent to the set }}$ $\left\{\left\langle\left\{(\ell, 3),(\ell, 4),\left(\ell^{\prime}, 4\right)\right\}, \emptyset\right\rangle\right\}$. In fact $\operatorname{Max}(L)$ is a union of $\preceq_{\text {univ-equivalent classes. Hence, }}$ the size of $\operatorname{Max}(L)$ can be reduced by keeping only one canonical element for each $\preceq_{\text {univ }}$ equivalent class. Given a set $s \in Q$, define its characteristic function $f_{s}:$ Loc $\rightarrow \mathbb{N} \cup\{\infty\}$ such that $f_{s}(\ell)=\inf \{n \mid(\ell, n) \in s\}$ with the usual convention that inf $\emptyset=\infty$. Note that if $f_{s}(\ell) \neq \infty$, then $f_{s}(\ell)$ is even for all $\ell \in \alpha$.

Let $f, g, f^{\prime}, g^{\prime}$ be characteristic functions. Let $\max \left(f, f^{\prime}\right)$ be the function $f^{\prime \prime}$ such that $f^{\prime \prime}(\ell)=\max \left\{f(\ell), f^{\prime}(\ell)\right\}$ for all $\ell \in$ Loc. We denote by $f_{\emptyset}$ the function such that $f_{\emptyset}(\ell)=\infty$ for all $\ell \in$ Loc. We write $f \leq f^{\prime}$ if for all $\ell \in$ Loc, $f(\ell) \leq f^{\prime}(\ell)$ and we write $\langle f, g\rangle \leq\left\langle f^{\prime}, g^{\prime}\right\rangle$ if $f \leq f^{\prime}, g \leq g^{\prime}$ and $g=f_{\emptyset}$ iff $g=f_{\emptyset}$. Notice that $\leq$ is partial order over characteristic functions, and that if $s \subseteq s^{\prime}$, then $f_{s^{\prime}} \leq f_{s}$ for all $s, s^{\prime} \in Q$. The following lemma is a corollary of Definition 5.1 .

Lemma 5.3. For all sets $s, s^{\prime}, o, o^{\prime} \in Q,\left\langle f_{s^{\prime}}, f_{o^{\prime}}\right\rangle \leq\left\langle f_{s}, f_{o}\right\rangle$ if and only if $\langle s, o\rangle \preceq$ univ $\left\langle s^{\prime}, o^{\prime}\right\rangle$.

Define $\llbracket f \rrbracket=\left\{s \in Q \mid \exists s^{\prime} \in Q: s \subseteq s^{\prime} \wedge f_{s^{\prime}}=f\right\}$ and $\llbracket\langle f, g\rangle \rrbracket=\left\{\langle s, o\rangle \mid\langle f, g\rangle \leq\left\langle f_{s}, f_{o}\right\rangle\right\}$. We extend the operator $\llbracket \cdot \rrbracket$ to sets of pairs of characteristic functions as expected. Notice 
that $f \leq f^{\prime}$ iff $\llbracket f^{\prime} \rrbracket \subseteq \llbracket f \rrbracket$, that $\llbracket \max \left(f, f^{\prime}\right) \rrbracket=\llbracket f \rrbracket \cap \llbracket f^{\prime} \rrbracket$, and a corollary of Lemma 5.3 is that the $\leq$-minimal elements of a set $L$ of pairs of characteristic functions represents exactly the

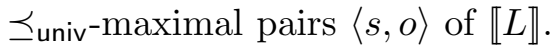

Now, we show how to compute efficiently $\cup, \cap$ and Pre for $\preceq$ univ-closed sets that are represented by characteristic functions. Let $L_{1}, L_{2}$ be two sets of pairs of characteristic functions, let $L_{\cup}$ be the set of $\leq$-minimal elements of $L_{1} \cup L_{2}$, and let $L_{\cap}$ be the $\leq$-minimal elements of the union of:

$\left\{\left\langle\max \left(f_{s}, f_{s^{\prime}}\right), \max \left(f_{o}, f_{o^{\prime}}\right)\right\rangle \mid\left\langle f_{s}, f_{o}\right\rangle \in L_{1} \wedge\left\langle f_{s^{\prime}}, f_{o^{\prime}}\right\rangle \in L_{2} \wedge \max \left(f_{o}, f_{o^{\prime}}\right) \neq f_{\emptyset}\right\}$ and $\left\{\left\langle\max \left(f_{s}, f_{s^{\prime}}\right), f_{\emptyset}\right\rangle \mid\left\langle f_{s}, f_{\emptyset}\right\rangle \in L_{1} \wedge\left\langle f_{s^{\prime}}, f_{\emptyset}\right\rangle \in L_{2}\right\}$.

By Equation (4.1) and by the previous remarks, we have:

$\llbracket L_{\cup} \rrbracket=\llbracket L_{1} \rrbracket \cup \llbracket L_{2} \rrbracket$ and $\llbracket L_{\cap} \rrbracket=\llbracket L_{1} \rrbracket \cap \llbracket L_{2} \rrbracket$.

To compute $\operatorname{Pre}_{\sigma}(\cdot)$ of a single pair of characteristic functions, we propose Algorithm 2 whose correctness is established by Theorem 5.4. Computing the predecessors of a set of characteristic functions is then straightforward using the algorithm for union of sets of pairs of characteristic functions since

$$
\operatorname{Pre}^{\mathrm{KVMH}(\mathcal{A})}(L)=\bigcup_{\sigma \in \Sigma} \bigcup_{\ell \in L} \operatorname{Pre}_{\sigma}^{\mathrm{KVMH}(\mathcal{A})}(\ell) .
$$

In Algorithm 2, we represent $\infty$ by any number strictly greater than $k=2(|\operatorname{Loc}|-|\alpha|)$, and we adapt the definition of $\leq$ as follows: $f \leq f^{\prime}$ iff for all $\ell \in$ Loc, either $f(\ell) \leq f^{\prime}(\ell)$ or $f^{\prime}(\ell)>k$. In the algorithm, we use the notations $\lceil n\rceil^{\text {odd }}$ for the least odd number $n^{\prime}$ such that $n^{\prime} \geq n$, and $\lceil n\rceil^{\text {even }}$ for the least even number $n^{\prime}$ such that $n^{\prime} \geq n$.

The structure of Algorithm 2 is similar to Algorithm 1, but the computations are expressed in terms of characteristic functions, thus in terms of ranks. For example, lines 4 [5 compute the equivalent of line 2 in Algorithm 1, where $\alpha_{1}$ corresponds here to the set of odd-ranked locations, and thus contains no $\alpha$-nodes. Details are given in the proof of Theorem 5.4.

Theorem 5.4. Let $\mathcal{A}=\langle$ Loc, $, \iota, \Sigma, \delta, \alpha\rangle$ be a $N B W, \sigma \in \Sigma$, and $\left\langle f_{s^{\prime}}, f_{o^{\prime}}\right\rangle$ be a pair of characteristic functions such that $f_{s^{\prime}} \leq f_{O^{\prime}}$. The set $L_{\text {Pre }}=\operatorname{Pre} \sigma_{\sigma}^{\text {univ }}\left(\left\langle f_{s^{\prime}}, f_{O^{\prime}}\right\rangle\right)$ computed by Algorithm 2 is such that $\llbracket L_{\mathrm{Pre}} \rrbracket=\operatorname{Pre}_{\sigma} \operatorname{KVMH}(\mathcal{A})\left(\llbracket\left\langle f_{s^{\prime}}, f_{o^{\prime}}\right\rangle \rrbracket\right)$ and for all $\left\langle f_{s}, f_{o}\right\rangle \in L_{\text {Pre }}$, we have $f_{s} \leq f_{o}$ and $f_{s}(\ell)$ and $f_{o}(\ell)$ are even for all $\ell \in \alpha$.

Proof. Let $\mathcal{A}^{c}=\operatorname{KVMH}(\mathcal{A})=\left\langle Q \times Q, q_{\iota}, \Sigma, \delta^{\prime}, \alpha^{\prime}\right\rangle$, and let $\left\langle s^{\prime}, o^{\prime}\right\rangle$ be a pair of sets whose characteristic functions are $\left\langle f_{s^{\prime}}, f_{o^{\prime}}\right\rangle$ and $o^{\prime} \subseteq s^{\prime}$ (such a pair exists because $f_{s^{\prime}} \leq f_{o^{\prime}}$ ). We show that (a) $\llbracket L_{\operatorname{Pre}} \rrbracket \subseteq \operatorname{Pre}_{\sigma}^{\mathcal{A}^{c}}\left(\llbracket\left\langle f_{s^{\prime}}, f_{o^{\prime}}\right\rangle \rrbracket\right)$ and (b) $\operatorname{Pre}_{\sigma}^{\mathcal{A}^{c}}\left(\llbracket\left\langle f_{s^{\prime}}, f_{o^{\prime}}\right\rangle \rrbracket\right) \subseteq \llbracket L_{\operatorname{Pre}} \rrbracket$.

To prove (a), first consider a pair $\left\langle f_{s}, f_{o}\right\rangle$ added to $L_{\text {Pre }}$ at line 13 of Algorithm 2 and let $\langle s, o\rangle \in \llbracket\left\langle f_{s}, f_{o}\right\rangle \rrbracket$. We show that $\langle s, o\rangle \stackrel{\sigma}{\rightarrow} \delta^{\prime}\left\langle s^{\prime}, o^{\prime}\right\rangle$ and $f_{s} \leq f_{o}$.

By the test of line 9, we have $f_{o} \neq f_{\emptyset}$ and therefore $o \neq \emptyset$. According to Definition 2.7 of $\operatorname{KVMH}(\mathcal{A})$, we have to check that there exists a set $o^{\prime \prime} \subseteq s^{\prime}$ such that $o^{\prime}=o^{\prime \prime} \backslash$ odd (we take $o^{\prime \prime}=o^{\prime} \cup\left(s^{\prime} \cap\right.$ odd $\left.)\right)$, and the following conditions hold:

(i) $\forall(\ell, n) \in s \cdot \forall \ell^{\prime} \in \delta(\ell, \sigma) \cdot \exists n^{\prime} \leq n:\left(\ell^{\prime}, n^{\prime}\right) \in s^{\prime}$.

Observe that for all $\ell \in$ Loc, for all $\ell^{\prime} \in \delta(\ell, \sigma)$, we have $f_{s^{\prime}}\left(\ell^{\prime}\right) \leq f_{s}(\ell)$ (lines 1112 of Algorithm 2). Since $f_{s}(\ell) \leq n$ (by definition of characteristic functions), we take $n^{\prime}=f_{s^{\prime}}\left(\ell^{\prime}\right)$ so that we have $n^{\prime} \leq f_{s}(\ell) \leq n$ and $\left(\ell^{\prime}, n^{\prime}\right) \in s^{\prime}$.

(ii) $\forall(\ell, n) \in o \cdot \forall \ell^{\prime} \in \delta(\ell, \sigma) \cdot \exists n^{\prime} \leq n:\left(\ell^{\prime}, n^{\prime}\right) \in o^{\prime \prime}$. 
Since $o^{\prime \prime}=o^{\prime} \cup\left(s^{\prime} \cap\right.$ odd $)$, we have $f_{o^{\prime \prime}}\left(\ell^{\prime}\right)=f_{o^{\prime}}\left(\ell^{\prime}\right)$ for $\ell^{\prime} \in \alpha$ and $f_{o^{\prime \prime}}\left(\ell^{\prime}\right)=$ $\min \left\{f_{o^{\prime}}\left(\ell^{\prime}\right),\left\lceil f_{s^{\prime}}\left(\ell^{\prime}\right)\right\rceil^{\text {odd }}\right\}$ for $\ell^{\prime} \notin \alpha$. Now, for all $\ell \in$ Loc, for all $\ell^{\prime} \in \delta(\ell, \sigma)$, we have either $\ell^{\prime} \in \alpha$ and then $f_{o}(\ell) \geq n^{\prime}$ for $n^{\prime}=f_{o^{\prime}}\left(\ell^{\prime}\right)$, or $\ell^{\prime} \notin \alpha$ and then $f_{o}(\ell) \geq n^{\prime}$ for $n^{\prime}=\min \left\{f_{o^{\prime}}\left(\ell^{\prime}\right),\left\lceil f_{s^{\prime}}\left(\ell^{\prime}\right)\right\rceil^{\text {odd }}\right\}$ (lines 4 -6 of Algorithm 2). In both cases, for $(\ell, n) \in o$ we have $f_{o^{\prime \prime}}\left(\ell^{\prime}\right) \leq n^{\prime} \leq f_{o}(\ell) \leq n$ and $\left(\ell^{\prime}, n^{\prime}\right) \in o^{\prime \prime}$.

Moreover, we prove that:

(iii) $f_{s} \leq f_{o}$.

Since $f_{s^{\prime}} \leq f_{o^{\prime}}$, we have for all $\ell^{\prime} \in$ Loc either $f_{o^{\prime}}\left(\ell^{\prime}\right)>k$ or $f_{o^{\prime}}\left(\ell^{\prime}\right) \geq f_{s^{\prime}}\left(\ell^{\prime}\right)$. By lines 4 [6 of Algorithm 2, we have for all $\ell \in$ Loc, for all $\ell^{\prime} \in \delta(\ell, \sigma)$ either $f_{o}(\ell) \geq f_{o^{\prime}}\left(\ell^{\prime}\right)$ or $f_{o}(\ell) \geq\left\lceil f_{s^{\prime}}\left(\ell^{\prime}\right)\right\rceil^{\text {odd }}$, and thus either $f_{o}(\ell)>k$ or $f_{o}(\ell) \geq f_{s^{\prime}}\left(\ell^{\prime}\right)$. Hence, we have for all $\ell \in$ Loc either $f_{o}(\ell)>k$ or $f_{o}(\ell) \geq \max \left\{f_{s^{\prime}}\left(\ell^{\prime}\right) \mid \ell^{\prime} \in \delta(\ell, \sigma)\right\}$. Therefore, by lines 11,12 of Algorithm 2, if $\ell \notin \alpha$, then $f_{o}(\ell)>k$ or $f_{o}(\ell) \geq f_{s}(\ell)$, and if $\ell \in \alpha$, then $f_{o}(\ell)$ is even (line 6) and thus either $f_{o}(\ell)>k$ or $f_{o}(\ell) \geq\left\lceil\max \left\{f_{s^{\prime}}\left(\ell^{\prime}\right) \mid \ell^{\prime} \in \delta(\ell, \sigma)\right\}\right.$ ]$^{\text {even }}=f_{s}(\ell)$. In all cases, $f_{s} \leq f_{o}$.

(iv) $\forall \ell \in \alpha: f_{s}(\ell)$ and $f_{o}(\ell)$ are even.

This is enforced by line 12 and line 6 of the algorithm.

Second, consider a pair $\left\langle f_{o}, f_{\emptyset}\right\rangle$ added to $L_{\text {Pre }}$ at line 7 , and let $\langle s, \emptyset\rangle \in \llbracket\left\langle f_{o}, f_{\emptyset}\right\rangle \rrbracket$. Notice that $f_{o} \leq f_{\emptyset}$ and that $f_{o}(\ell)$ is even for all $\ell \in \alpha$ by $(i v)$. We show that there exists $\left\langle s^{\prime \prime}, o^{\prime \prime}\right\rangle \preceq_{\text {univ }}$ $\left\langle s^{\prime}, o^{\prime}\right\rangle$ such that $\langle s, \emptyset\rangle \stackrel{\sigma}{\rightarrow}_{\delta^{\prime}}\left\langle s^{\prime \prime}, o^{\prime \prime}\right\rangle$. We take $s^{\prime \prime}=o^{\prime} \cup\left(s^{\prime} \cap\right.$ odd $)$ and $o^{\prime \prime}=s^{\prime \prime} \backslash$ odd. Since $o^{\prime} \subseteq s^{\prime}$, we have (1) $s^{\prime \prime} \subseteq s^{\prime}$, and we have (2) $o^{\prime \prime}=o^{\prime} \backslash$ odd $\subseteq o^{\prime}$. Moreover, if $o^{\prime} \neq \emptyset$, then there exists let $(\ell, n) \in o^{\prime}$ for some $\ell \in$ Loc and even number $n$, since the maximal rank $k=2(|\operatorname{Loc}|-|\alpha|)$ is even. So $(\ell, n) \in o^{\prime \prime}$ and thus $o^{\prime \prime} \neq \emptyset$. Since $o^{\prime \prime} \subseteq o^{\prime}$, we have (3) $o^{\prime} \neq \emptyset$ iff $o^{\prime \prime} \neq \emptyset$. Hence $\left\langle s^{\prime \prime}, o^{\prime \prime}\right\rangle \preceq$ univ $\left\langle s^{\prime}, o^{\prime}\right\rangle$. The fact that $\left\langle f_{o}, \emptyset\right\rangle \stackrel{\sigma}{\rightarrow} \delta^{\prime}\left\langle s^{\prime \prime}, o^{\prime \prime}\right\rangle$ is proven similarly to $(i i)$.

To prove (b), assume that there exist $\left\langle s_{1}, o_{1}\right\rangle$ and $\left\langle s_{1}^{\prime}, o_{1}^{\prime}\right\rangle$ such that $\left\langle s_{1}, o_{1}\right\rangle \stackrel{\sigma}{\rightarrow} \delta_{\delta^{\prime}}\left\langle s_{1}^{\prime}, o_{1}^{\prime}\right\rangle$ and $\left\langle s_{1}^{\prime}, o_{1}^{\prime}\right\rangle \in \llbracket\left\langle f_{s^{\prime}}, f_{o^{\prime}}\right\rangle \rrbracket$. We have to show that $\left\langle s_{1}, o_{1}\right\rangle \in \llbracket L_{\text {Pre }} \rrbracket$, i.e., $\left\langle f_{s_{1}}, f_{o_{1}}\right\rangle \geq\left\langle f_{s}, f_{o}\right\rangle$ for some $\left\langle f_{s}, f_{o}\right\rangle \in L_{\text {Pre }}$.

First, assume that $o_{1} \neq \emptyset$. Notice that $f_{s_{1}^{\prime}} \geq f_{s^{\prime}}$ and $f_{o_{1}^{\prime}} \geq f_{o^{\prime}}$ since $\left\langle s_{1}^{\prime}, o_{1}^{\prime}\right\rangle \in \llbracket\left\langle f_{s^{\prime}}, f_{o^{\prime}}\right\rangle \rrbracket$, From the fact that $\left\langle s_{1}, o_{1}\right\rangle \stackrel{\sigma}{\rightarrow}_{\delta^{\prime}}\left\langle s_{1}^{\prime}, o_{1}^{\prime}\right\rangle$, we get:

(i) for all $\left(\ell, n_{1}\right) \in s_{1}$, for all $\ell^{\prime} \in \delta(\ell, \sigma), n_{1} \geq f_{s_{1}}(\ell) \geq f_{s_{1}^{\prime}}\left(\ell^{\prime}\right)$ and thus $n_{1} \geq f_{s^{\prime}}\left(\ell^{\prime}\right)$. Hence, for all $\ell \in$ Loc we have $f_{s_{1}}(\ell) \geq \max \left\{f_{s^{\prime}}\left(\ell^{\prime}\right) \mid \ell^{\prime} \in \delta(\ell, \sigma)\right\}=f_{s}(\ell)$, where $f_{s}$ is computed by line 11 of Algorithm 2) for $\ell \notin \alpha$. We also have $f_{s_{1}}(\ell) \geq f_{s}(\ell)$ (see line 12 of Algorithm 2) for $\ell \in \alpha$, as $f_{s_{1}}(\ell)$ is even in that case. Thus, $f_{s} \leq f_{s_{1}}$.

(ii) for all $\left(\ell, n_{2}\right) \in o_{1}$, for all $\ell^{\prime} \in \delta(\ell, \sigma), n_{2} \geq f_{o_{1}}(\ell) \geq f_{o_{1}^{\prime \prime}}\left(\ell^{\prime}\right)$ for some set $o_{1}^{\prime \prime}$ such that $o_{1}^{\prime \prime} \subseteq s_{1}^{\prime}$ and $o_{1}^{\prime \prime} \backslash$ odd $=o_{1}^{\prime}$. Therefore $o_{1}^{\prime \prime} \subseteq o_{1}^{\prime} \cup\left(s_{1}^{\prime} \cap\right.$ odd $)$ and thus $f_{o_{1}^{\prime \prime}} \geq f_{o_{1}^{\prime} \cup\left(s_{1}^{\prime} \text { กodd }\right)} \geq$ $f_{o^{\prime} \cup\left(s^{\prime} \cap \text { odd }\right)}$ since $f_{s_{1}^{\prime}} \geq f_{s^{\prime}}$ and $f_{o_{1}^{\prime}} \geq f_{o^{\prime}}$. Hence, for all $\ell \in$ Loc either $f_{o_{1}}(\ell)>k$ or $f_{o_{1}}(\ell) \geq f_{o}(\ell)$ (where $f_{o}$ is computed at lines [1] of Algorithm 2). Thus, $f_{o} \leq f_{o_{1}}$.

(iii) By our assumption that $o_{1} \neq \emptyset$, we have $f_{o_{1}} \neq f_{\emptyset}$, and so $f_{o} \neq f_{\emptyset}$ by $(i i)$.

Hence, the pair $\left\langle f_{s}, f_{o}\right\rangle$ added to $L_{\text {Pre }}$ by Algorithm 2 at line 13 satisfies $\left\langle f_{s_{1}}, f_{o_{1}}\right\rangle \geq\left\langle f_{s}, f_{o}\right\rangle$ and thus $\left\langle s_{1}, o_{1}\right\rangle \in \llbracket L_{\mathrm{Pre}} \rrbracket$.

Second, assume that $o_{1}=\emptyset$. Let $s^{\prime \prime}=o^{\prime} \cup\left(s^{\prime} \cap\right.$ odd $)$. Since $\left\langle s_{1}, o_{1}\right\rangle \stackrel{\sigma}{\rightarrow} \delta_{\delta^{\prime}}\left\langle s_{1}^{\prime}, o_{1}^{\prime}\right\rangle$ and $o_{1}=\emptyset$, we have $o_{1}^{\prime}=s_{1}^{\prime} \backslash$ odd. Next, we use several times the fact that $u \subseteq v$ implies $f_{v} \leq f_{u}$. Since $f_{s_{1}^{\prime}} \geq f_{s^{\prime}}$ and $f_{o_{1}^{\prime}} \geq f_{o^{\prime}}$, we have (1) $f_{s_{1}^{\prime} \text { Пodd }} \geq f_{s^{\prime} \text { nodd }} \geq f_{s^{\prime \prime}}$ and (2) $f_{s_{1}^{\prime} \text { \odd }}=f_{o_{1}^{\prime}} \geq f_{o^{\prime}} \geq f_{s^{\prime \prime}}$. By (1) and (2), we get easily $f_{s_{1}^{\prime}} \geq f_{s^{\prime \prime}}$. Now, by the fact that 


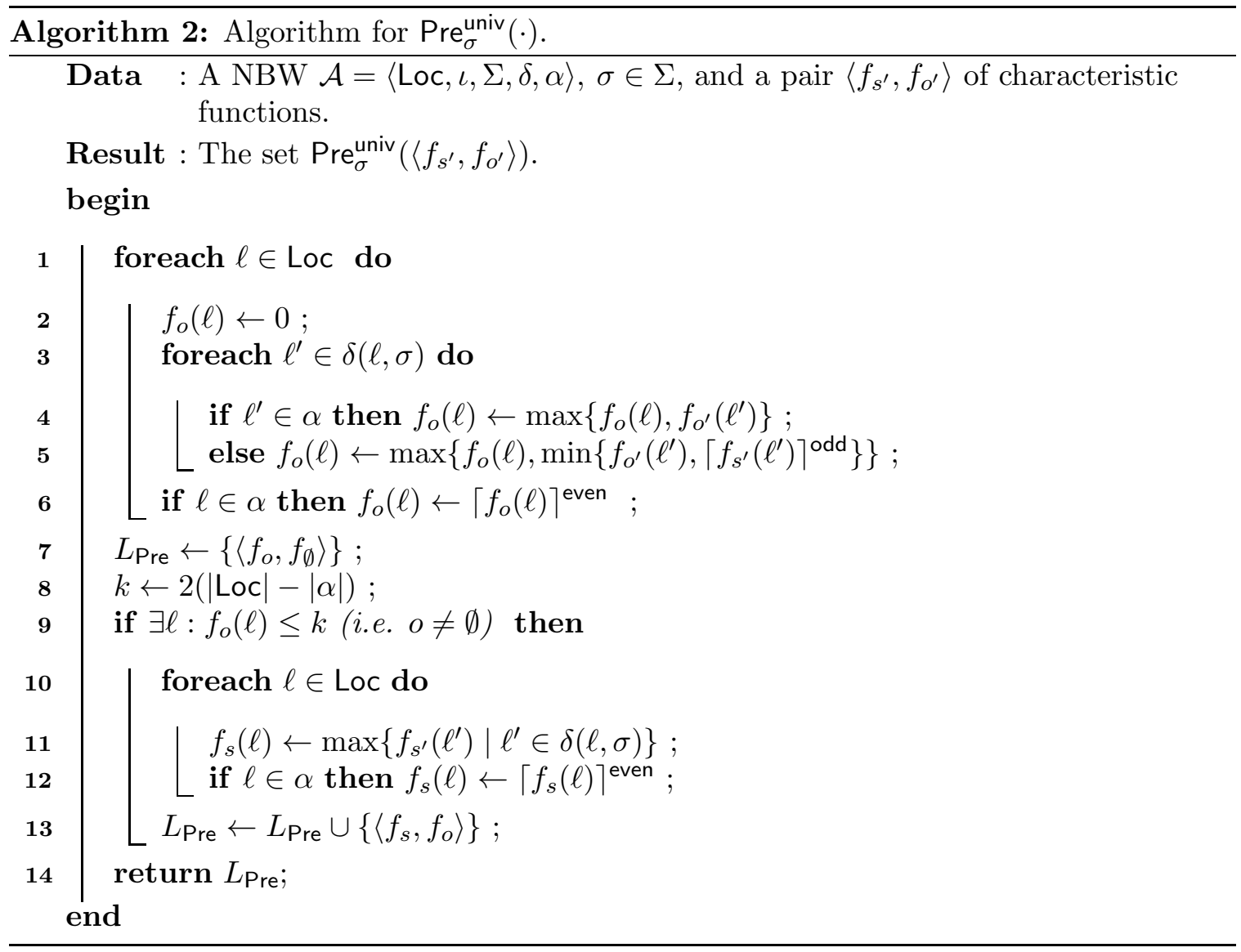

$\left\langle s_{1}, o_{1}\right\rangle \stackrel{\sigma}{\rightarrow}_{\delta^{\prime}}\left\langle s_{1}^{\prime}, o_{1}^{\prime}\right\rangle$, we know that for all $\left(\ell, n_{1}\right) \in s_{1}$, for all $\ell^{\prime} \in \delta(\ell, \sigma), n_{1} \geq f_{s_{1}^{\prime}}\left(\ell^{\prime}\right)$ and thus $n_{1} \geq f_{s^{\prime \prime}}\left(\ell^{\prime}\right)$. Notice that $f_{o}(\ell)=\max \left\{f_{s^{\prime \prime}}\left(\ell^{\prime}\right) \mid \ell^{\prime} \in \delta(\ell, \sigma)\right\}$, where $f_{o}$ is computed at lines 116 of Algorithm 2. Thus, $n_{1} \geq f_{o}(\ell)$ for all $\ell \in$ Loc and therefore $f_{s_{1}} \leq f_{o}$ so that $\left\langle s_{1}, o_{1}\right\rangle \in \llbracket\left\langle f_{o}, f_{\emptyset}\right\rangle \rrbracket$ where $\left\langle f_{o}, f_{\emptyset}\right\rangle$ is added to $L_{\text {Pre }}$ by Algorithm 2 at line 13.

Algorithm 2 computes the predecessors of a pair $\left\langle f_{s^{\prime}}, f_{o^{\prime}}\right\rangle$ in time $O\left(\mid\right.$ Loc $\left.\left.\right|^{2}\right)$, which is polynomial in the size of the input, even though the number of pairs $\left\langle s^{\prime}, o^{\prime}\right\rangle$ that are represented by the pair $\left\langle f_{s^{\prime}}, f_{o^{\prime}}\right\rangle$ and by the computed set $L_{\text {Pre }}$ can be of exponential size. For example, the set $\alpha^{\prime}=Q \times\{\emptyset\}$ with an exponential number of elements is represented by the unique pair $\left\langle f_{s}, f_{\emptyset}\right\rangle$ where $f_{s}(\ell)=0$ for all $\ell \in$ Loc. Hence the compact representation that we propose does not come with an execution time blow-up, which makes the new approach much more efficient in practice.

\section{Implementation AND Practical Evaluation}

The randomized model. To evaluate our new algorithm for universality of NBW and compare with the existing implementations of the Kupferman-Vardi and Miyano-Hayashi constructions, we use a random model to generate NBW. This model was first proposed by Tabakov and Vardi to compare the efficiency of some algorithms for automata in the context of finite words automata [TV05] and more recently in the context of infinite words 
automata [TV07]. In the model, the input alphabet is fixed to $\Sigma=\{0,1\}$, and for each letter $\sigma \in \Sigma$, a number $k_{\sigma}$ of different state pairs $\left(\ell, \ell^{\prime}\right) \in \operatorname{Loc} \times$ Loc are chosen uniformly at random before the corresponding transitions $\left(\ell, \sigma, \ell^{\prime}\right)$ are added to the automaton. The ratio $r_{\sigma}=\frac{k_{\sigma}}{\mid \text { Loc } \mid}$ is called the transition density for $\sigma$. This ratio represents the average outdegree of each state for $\sigma$. In all experiments, we choose $r_{0}=r_{1}$, and denote the transition density by $r$. The model contains a second parameter: the density $f$ of accepting states. There is only one initial state, and the number $m$ of accepting states is linear in the total number of states, as determined by $f=\frac{m}{|\operatorname{Loc}|}$. The accepting states themselves are chosen uniformly at random. Observe that since the transition relation is not always total, automata with $f=1$ are not necessarily universal.

Tabakov and Vardi have studied the space of parameter values for this model and argue that "interesting" automata are generated by the model as the two parameters $r$ and $f$ vary. They also study the density of universal automata.

Performance comparison. We have implemented our algorithm to check the universality of randomly generated NBW. The code is written in $\mathrm{C}$ with an explicit representation for characteristic functions, as arrays of integers. All the experiments are conducted on a biprocessor Linux station (two 3.06Ghz Intel Xeons with 4GB of RAM).

Figure 3 shows as a function of $r$ (transition density) and $f$ (density of accepting states) the median execution times for testing universality of 100 random automata with $\mid$ Loc $\mid=30$. It shows that the universality test was the most difficult for $r=1.8$ and $f=0.1$ with a median time of 11 seconds. The times for $r \leq 1$ and $r \geq 2.8$ are not plotted because they were always less than $250 \mathrm{~ms}$. A similar shape and maximal median time is reported by Tabakov for automata of size 6, that is for automata that are five times smaller [TV07]. Another previous work reports prohibitive execution times for complementing NBW of size 6 , showing that explicitly constructing the complement is not a reasonable approach GKSV03. The density of universal automata in the samples is shown in Figure 4. The density increases when states have more transitions, while it seems less sensitive to the density of accepting states. The difficult instances correspond to the values of the densities of transitions and accepting states for which the probability to be universal is close to a half. Analogous results have been observed in [TV07.

To evaluate the scalability of our algorithm, we have run the following experiment. For a set of parameter values, we have evaluated the maximal size of automata (measured in term of number of locations) for which our algorithm could analyze 50 over 100 instances in less than 20 seconds. We have tried automata sizes from 10 to 1500, with a fine granularity for small sizes (from 10 to 100 with an increment of 10, from 100 to 200 with an increment of 20, and from 200 to 500 with an increment of 30) and a rougher granularity for large sizes (from 500 to 1000 with an increment of 50, and from 1000 to 1500 with an increment of 100).

The results are shown in Fig. [5, and the corresponding values are given in Table 1, The vertical scale is logarithmic. For example, for $r=2$ and $f=0.5$, our algorithm was able to handle at least 50 automata of size 120 in less than 20 seconds and was not able to do so for automata of size 140. In comparison, Tabakov and Vardi have studied the behavior of Kupferman-Vardi and Miyano-Hayashi constructions for different implementation schemes. We compare with the performances of their symbolic approach which is the most efficient. For the same parameter values $(r=2$ and $f=0.5)$, they report that their implementation can handle NBW with at most 8 states in less than 20 seconds [TV07]. 


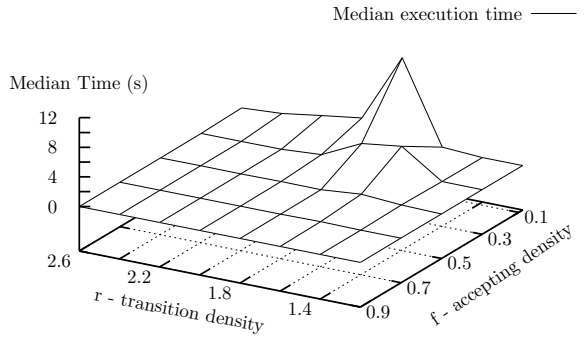

Figure 3: Median time to check universality of 100 automata of size 30 for each sample point.

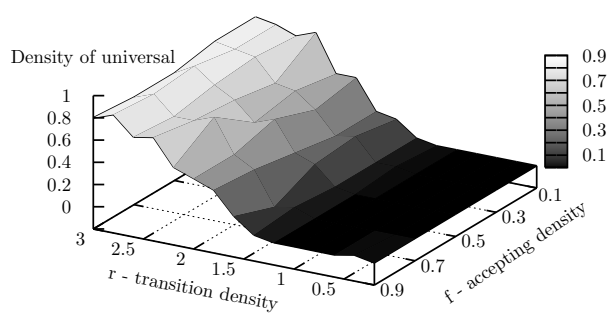

Figure 4: Density of universal automata for the samples of Figure 3 .

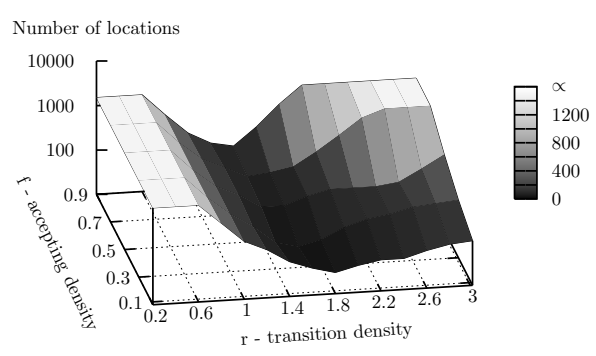

Figure 5: Automata size for which the median execution time to check universality is less than 20 seconds (log scale). See also Table 1 .

Table 1: Automata size (NBW) for which the median execution time for checking universality is less than 20 seconds. The symbol $\propto$ means more than 1500 .

\begin{tabular}{|c||c|c|c|c|c|c|c|c|c|c|c|c|c|c|c|}
\hline $\mathrm{f}$ & 0.2 & 0.4 & 0.6 & 0.8 & 1.0 & 1.2 & 1.4 & 1.6 & 1.8 & 2.0 & 2.2 & 2.4 & 2.6 & 2.8 & 3.0 \\
\hline \hline 0.1 & $\propto$ & $\propto$ & $\propto$ & 550 & 200 & 120 & 60 & 40 & 30 & 40 & 50 & 50 & 70 & 90 & 100 \\
\hline 0.3 & $\propto$ & $\propto$ & $\propto$ & 500 & 200 & 100 & 40 & 30 & 40 & 70 & 100 & 120 & 160 & 180 & 200 \\
\hline 0.5 & $\propto$ & $\propto$ & $\propto$ & 500 & 200 & 120 & 60 & 60 & 90 & 120 & 120 & 120 & 140 & 260 & 500 \\
\hline 0.7 & $\propto$ & $\propto$ & $\propto$ & 500 & 200 & 120 & 70 & 80 & 100 & 200 & 440 & 1000 & $\propto$ & $\propto$ & $\propto$ \\
\hline 0.9 & $\propto$ & $\propto$ & $\propto$ & 500 & 180 & 100 & 80 & 200 & 600 & $\propto$ & $\propto$ & $\propto$ & $\propto$ & $\propto$ & $\propto$ \\
\hline
\end{tabular}

In Figure 6, we show the median execution time to check universality for relatively difficult instances $(r=2$ and $f$ vary from 0.3 to 0.7$)$. The vertical scale is logarithmic, so the behavior is roughly exponential in the size of the automata. Similar analyzes are reported in [TV07] but for sizes below 10.

Finally, we give in Figure 7 the distribution of execution times for 100 automata of size 50 with $r=2.2$ and $f=0.5$, so that roughly half of the instances are universal. Each point represents one automaton, and one point lies outside the figure with an execution 


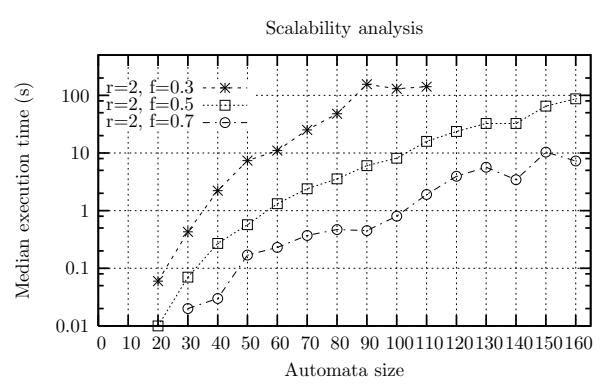

Figure 6: Median time to check universality (of 100 automata for each sample point).

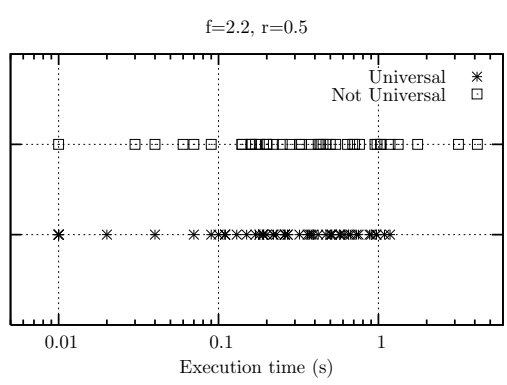

Figure 7: Execution time to check universality of 100 automata, 57 of which were universal.

time of $675 \mathrm{~s}$ for a non universal automaton. The existence of very few instances that are very hard was often encountered in the experiments, and this is why we use the median for the execution times. If we except this hard instance, Figure 7 shows that universal automata (average time $350 \mathrm{~ms}$ ) are slightly easier to analyze than non-universal automata (average time $490 \mathrm{~ms}$ ). This probably comes from the fact that we stop the computation of the (greatest) fixed point whenever the initial state is not in the $\preceq$ univ-closure of the current approximation. Indeed, in such case, since the approximations are $\preceq_{\text {univ-decreasing, we }}$ know that the initial state would also not lie in the fixed point. Of course, this optimization applies only for universal automata.

\section{LANGUAGE INCLUSION FOR BÜCHI AUTOMATA}

Let $\mathcal{A}_{1}=\left\langle\operatorname{Loc}_{1}, \iota_{1}, \Sigma, \delta_{1}, \alpha_{1}\right\rangle$ and $\mathcal{A}_{2}$ be two NBW defined on the same alphabet $\Sigma$ for which we want to check language inclusion: $\mathcal{L}\left(\mathcal{A}_{1}\right) \subseteq$ ? $\mathcal{L}\left(\mathcal{A}_{2}\right)$. To solve this problem, we check emptiness of $\mathcal{L}\left(\mathcal{A}_{1}\right) \cap \mathcal{L}^{c}\left(\mathcal{A}_{2}\right)$. As we have seen, we can use the Kupferman-Vardi and Miyano-Hayashi construction to specify a NBW $\mathcal{A}_{2}^{c}=\left\langle\operatorname{Loc}_{2}, \iota_{2}, \Sigma, \delta_{2}, \alpha_{2}\right\rangle$ that accepts the complement of the language of $\mathcal{A}_{2}$.

Using the classical product construction, let $\mathcal{B}=\mathcal{A}_{1} \times \mathcal{A}_{2}^{c}$ be a finite automaton with set of locations $\operatorname{Loc}_{\mathcal{B}}=\operatorname{Loc}_{1} \times \operatorname{Loc}_{2}$, initial state $\iota_{\mathcal{B}}=\left(\iota_{1}, \iota_{2}\right)$, and transition function $\delta_{\mathcal{B}}$ such that $\delta_{\mathcal{B}}\left(\left(\ell_{1}, \ell_{2}\right), \sigma\right)=\delta_{1}\left(\ell_{1}, \sigma\right) \times \delta_{2}\left(\ell_{2}, \sigma\right)$. We equip $\mathcal{B}$ with the generalized Büchi condition $\left\{\beta_{1}, \beta_{2}\right\}=\left\{\alpha_{1} \times \operatorname{Loc}_{2}\right.$, $\left.\operatorname{Loc}_{1} \times \alpha_{2}\right\}$, thus asking for a run of $\mathcal{B}$ to be accepting that it visits $\beta_{1}$ and $\beta_{2}$ infinitely often. It is routine to show that we have $\mathcal{L}(\mathcal{B})=\mathcal{L}\left(\mathcal{A}_{1}\right) \cap \mathcal{L}\left(\mathcal{A}_{2}^{c}\right)$. The following fixed point

$$
\mathcal{F}_{\mathcal{B}}^{\prime} \equiv \nu y \cdot\left(\mu x_{1} \cdot\left[\operatorname{Pre}^{\mathcal{B}}\left(x_{1}\right) \cup\left(\operatorname{Pre}^{\mathcal{B}}(y) \cap \beta_{1}\right)\right] \cap \mu x_{2} \cdot\left[\operatorname{Pre}^{\mathcal{B}}\left(x_{2}\right) \cup\left(\operatorname{Pre}^{\mathcal{B}}(y) \cap \beta_{2}\right)\right]\right)
$$

can be used to check emptiness of $\mathcal{B}$ as we have $\mathcal{L}(\mathcal{B}) \neq \emptyset$ iff $\iota_{\mathcal{B}} \in \mathcal{F}_{\mathcal{B}}^{\prime}$. We now define the pre-order $\preceq_{\text {inc }}$ over the locations of $\mathcal{B}$ : for all $\left(\ell_{1}, \ell_{2}\right),\left(\ell_{1}^{\prime}, \ell_{2}^{\prime}\right) \in \operatorname{Loc} \mathcal{B}$, let $\left(\ell_{1}, \ell_{2}\right) \preceq_{\text {inc }}\left(\ell_{1}^{\prime}, \ell_{2}^{\prime}\right)$ iff $\ell_{1}=\ell_{1}^{\prime}$ and $\ell_{2} \preceq_{\text {univ }} \ell_{2}^{\prime}$.

We extend the definition of simulation relation $\preceq$ (Definition 3.1) to generalized Büchi automata $\mathcal{B}$ by asking that for each $\beta_{i}$, the relation $\preceq$ is a simulation for $\mathcal{B}$ with accepting states $\beta_{i}$.

Lemma 7.1. The relation $\preceq_{\text {inc }}$ is a simulation for $\mathcal{B}$. 
Proof. First, observe that equality is a simulation relation for $\mathcal{A}_{1}$. Then, the first condition of Definition 3.1 is a direct consequence of the fact that equality (resp. $\preceq$ univ ) is a simulation relation for $\mathcal{A}_{1}$ (resp. for $\mathcal{A}_{2}^{c}$ ), and that $\mathcal{B}=\mathcal{A}_{1} \times \mathcal{A}_{2}^{c}$ is the product of these automata. Second, it is easy to see that the sets $\beta_{1}$ and $\beta_{2}$ are $\preceq_{\text {inc-closed. }}$

As a consequence of the last lemma, we know that all sets that we have to manipulate to solve the language inclusion problem using the fixed point $\mathcal{F}_{\mathcal{B}}^{\prime}$ are $\preceq$ inc-closed. The operators $\cup, \cap$ and Pre can be thus computed efficiently, using the same algorithms and data structures as for universality. In particular, let $\operatorname{Pre}_{\sigma}^{\text {inc }}\left(\ell_{1}^{\prime}, \ell_{2}^{\prime}\right)=\operatorname{Pre}_{\sigma}^{\mathcal{A}_{1}}\left(\ell_{1}^{\prime}\right) \times \operatorname{Pre}_{\sigma}^{\text {univ }}\left(\ell_{2}^{\prime}\right)$ where Pre $\sigma_{\sigma}^{\text {univ }}$ is computed by Algorithm 2 (with input $\mathcal{A}_{2}$ ). It is easy to show as a corollary of Theorem 5.4 that $\downarrow \operatorname{Pre}_{\sigma}^{\text {inc }}\left(\ell_{1}^{\prime}, \ell_{2}^{\prime}\right)=\operatorname{Pre}_{\sigma}^{\mathcal{B}}\left(\downarrow\left\{\left(\ell_{1}^{\prime}, \ell_{2}^{\prime}\right)\right\}\right)$.

\section{Conclusion}

We have shown that the prohibitive complementation constructions for nondeterministic Büchi automata can be avoided for solving classical problems like universality and language inclusion. Our approach is based on fixed points computation and the existence of simulation relations for the (exponential) constructions used in complementation of Büchi automata. Those simulations are used to dramatically reduce the amount of computations needed to decide classical problems. Their definition relies on the structure of the original automaton and do not require explicit complementation.

The resulting algorithms evaluate a fixed point formula and avoid redundant computations by maintaining sets of maximal elements according to the simulation relation. In practice, the computation of the predecessor operator, which is the key of the approach, is efficient because it is done on antichains of elements only. Even though the classical approaches (as well as ours) have the same worst case complexity, our prototype implementation outperforms those approaches where the structural properties of the complement automaton (witnessed by the existence of simulation relations) is not exploited. The huge gap of performances holds over the entire parameter space of the randomized model proposed by Tabakov and Vardi.

Applications of this paper go beyond universality and language inclusion for NBW, as we have shown that the methodology applies to alternating Büchi automata for which efficient translations from LTL formula are known [GO01]. Significant improvements in the modelchecking and satisfiability problem of LTL can be achieved with the same ideas DDMR08b, DDMR08a.

Acknowledgment. We thank two anonymous reviewers for helpful comments and suggestions.

\section{REFERENCES}

[BGS00] R. Bloem, H. N. Gabow, and F. Somenzi. An algorithm for strongly connected component analysis in $\log$ symbolic steps. In Proceedings of FMCAD: Formal Methods in ComputerAided Design, Lecture Notes in Computer Science 1954, pages 37-54. Springer, 2000.

[Büc62] J. Richard Büchi. On a decision method in restricted second order arithmetic. In Proc. International Congress on Logic, Method, and Philosophy of Science, pages 1-12. Stanford University Press, 1962.

[CDHR07] K. Chatterjee, L. Doyen, T. A. Henzinger, and J.-F. Raskin. Algorithms for omega-regular games of incomplete information. Logical Methods in Computer Science, 3(3:4), 2007. 
[DDHR06] M. De Wulf, L. Doyen, T. A. Henzinger, and J.-F. Raskin. Antichains: A new algorithm for checking universality of finite automata. In Proceedings of CAV: Computer-Aided Verification, Lecture Notes in Computer Science 4144, pages 17-30. Springer-Verlag, 2006.

[DDMR08a] M. De Wulf, L. Doyen, N. Maquet, and J.-F. Raskin. Alaska: Antichains for logic, automata and symbolic kripke structures analysis. In Proceedings of ATVA 2008: Automated Technology for Verification and Analysis, Lecture Notes in Computer Science 5311, pages 240-245. SpringerVerlag, 2008.

[DDMR08b] M. De Wulf, L. Doyen, N. Maquet, and J.-F. Raskin. Antichains: Alternative algorithms for LTL satisfiability and model-checking. In Proceedings of TACAS: Tools and Algorithms for the Construction and Analysis of Systems, Lecture Notes in Computer Science 4963, pages 63-77. Springer-Verlag, 2008.

[DDR06] M. De Wulf, L. Doyen, and J.-F. Raskin. A lattice theory for solving games of imperfect information. In Proceedings of HSCC: Hybrid Systems-Computation and Control, Lecture Notes in Computer Science 3927, pages 153-168. Springer-Verlag, 2006.

[EL86] E. A. Emerson and C.-L. Lei. Efficient model checking in fragments of the propositional mucalculus (extended abstract). In Proceedings of LICS: Symposium on Logic in Computer Science, pages 267-278. IEEE Computer Society, 1986.

[EWS05] K. Etessami, T. Wilke, and R. A. Schuller. Fair simulation relations, parity games, and state space reduction for Büchi automata. SIAM J. Comput., 34(5):1159-1175, 2005.

[GKSV03] S. Gurumurthy, O. Kupferman, F. Somenzi, and M. Y. Vardi. On complementing nondeterministic Büchi automata. In Proceedings of CHARME: Correct Hardware Design and Verification Methods, Lecture Notes in Computer Science 2860, pages 96-110. Springer-Verlag, 2003.

[GO01] P. Gastin and D. Oddoux. Fast LTL to Büchi automata translation. In Proceedings of CAV: Computer-Aided Verification, Lecture Notes in Computer Science 2102, pages 53-65. SpringerVerlag, 2001.

[GPP03] R. Gentilini, C. Piazza, and A. Policriti. Computing strongly connected components in a linear number of symbolic steps. In Proceedings of SODA: ACM-SIAM Symposium on Discrete Algorithms, pages 573-582, 2003.

[KV01] O. Kupferman and M. Y. Vardi. Weak alternating automata are not that weak. ACM Trans. Comput. Log., 2(3):408-429, 2001.

[MH84] S. Miyano and T. Hayashi. Alternating finite automata on omega-words. In Proceedings of CAAP: Int. Colloquium on Trees in Algebra and Programming, pages 195-210, 1984.

[Mic88] M. Michel. Complementation is more difficult with automata on infinite words. CNET, Paris, 1988.

[RBS00] K. Ravi, R. Bloem, and F. Somenzi. A comparative study of symbolic algorithms for the computation of fair cycles. In Proceedings of FMCAD: Formal Methods in Computer-Aided Design, Lecture Notes in Computer Science 1954, pages 143-160. Springer, 2000.

[RH04] T. C. Ruys and G. J. Holzmann. Advanced spin tutorial. In SPIN, Lecture Notes in Computer Science 2989, pages 304-305. Springer-Verlag, 2004.

[Saf88] S. Safra. On the complexity of $\omega$-automata. In Proceedings of FOCS: Foundations of Computer Science, pages 319-327. IEEE, 1988.

[SVW87] A. P. Sistla, M. Y. Vardi, and P. Wolper. The complementation problem for Büchi automata with applications to temporal logic. Theor. Comput. Sci., 49:217-237, 1987.

[TV05] D. Tabakov and M. Y. Vardi. Experimental evaluation of classical automata constructions. In Proceedings of LPAR: Logic for Programming, Artificial Intelligence, and Reasoning, Lecture Notes in Computer Science 3835, pages 396-411. Springer-Verlag, 2005.

[TV07] D. Tabakov and M. Y. Vardi. Model-checking Büchi specifications. In Pre-proceedings of LATA: Language and Automata Theory and Applications, 2007.

[VW86] M. Y. Vardi and P. Wolper. An automata-theoretic approach to automatic program verification (preliminary report). In Proceedings of LICS: Symposium on Logic in Computer Science, pages 332-344. IEEE Computer Society, 1986.

[VW94] M. Y. Vardi and P. Wolper. Reasoning about infinite computations. Inf. Comput., 115(1):1-37, 1994. 